\title{
Enseigner le droit à l'Université populaire lyonnaise, entre activité doctrinale et engagement politique (1899-1903)
}

\begin{abstract}
J. Henning, «Enseigner le droit à l'Université populaire lyonnaise, entre activité doctrinale et engagement politique (1899-1903) », in Les Études sociales, 2021/1, n 173, p. 51-89
\end{abstract}

Cairn : https://www.cairn.info/revue-les-etudes-sociales-2021-1.htm

Les Études sociales : http://lasciencesociale.org/20211-(n\%c2\%b0-173).html

\begin{abstract}
"Bâtissons la Maison du Peuple en équité! Ayons la loi pour fil et le droit pour équerre ; Choisissons la Raison comme première pierre. Pour que les fondements durent l'éternité !» 1
\end{abstract}

En novembre 1900, c'est par ces quelques vers aux accents maçonniques qu'est inaugurée l'Université populaire lyonnaise, rue Hénon, dans le quartier de la Croix-Rousse. Dans une grande messe républicaine, les dreyfusards lyonnais se réunissent autour de la fondatrice de cette nouvelle Université populaire, Jeanne Desparmet-Ruello. Parmi eux, figurent des notables comme le maire Victor Augagneur, le botaniste et futur sénateur Georges Beauvisage, le futur président du conseil Édouard Herriot - alors seulement professeur au Lycée Ampère - et certains grands noms de la Faculté de droit : Louis Josserand, Jean Appleton, Paul $\mathrm{Pic}^{2}$. La présence de ces professeurs questionne immédiatement la place que peut occuper le droit dans l'éducation populaire pour adultes au tournant du $\mathrm{XX}^{\mathrm{e}}$ siècle.

À bien des égards, le concept d'Université populaire se range dans la catégorie plus large de l'éducation populaire entendue «comme l'action éducative qui prétend toucher principalement les milieux populaires et qui entend agir sur l'individu, en particulier la jeunesse, hors de l'école pour transformer la société » ${ }^{3}$. En cela, l'éducation populaire revêt une portée politique dès lors qu'elle rencontre, à la fin du siècle, le projet républicain : acculturer la population à la République libérale et démocratique ${ }^{4}$. Toutefois, l'Université populaire constitue une expérience pédagogique à part en raison de l'esprit qui l'anime et du public auquel elle s'adresse ${ }^{5}$. Formée dans les dernières années du XIX ${ }^{\mathrm{e}}$ siècle, les Universités populaires sont établies en France dans un esprit éducatif et non professionnel. Elles se distinguent alors d'autres formes d'éducation populaire déjà existantes, comme les bibliothèques populaires ${ }^{6}$ et

\footnotetext{
${ }^{1}$ F. Gregh, La maison du peuple, in La beauté de vivre, Paris, Calmann Lévy, 1901, p. 208.

2 Annales de l'Université populaire lyonnaise, $\mathrm{n}^{\circ} 1, \mathrm{p} .19$.

${ }^{3}$ C. Christen et C. Fayolle, «Écoles du peuple, Écoles des pauvres ? », in Revue d'histoire du XIX ${ }^{e}$ siècle, $\mathrm{n}^{\circ} 55$, 2017 , p. 22. La détermination du domaine de l'éducation populaire donne lieu à des débats historiographiques : F. Tétard, «De l'affaire Dreyfus à la guerre d'Algérie, un siècle d'éducation populaire », in Esprit, mars 2002, p. 39-59 ; C. Christen et L. Besse (dir.), Histoire de l'éducation populaire, 1815-1945, Villeneuve d'Ascq, Presses universitaires du Septentrion, 2017, p. 11-50 ; C. Christen, «Jalons pour une histoire de l'éducation industrielle destinée aux ouvriers adultes au XIX ${ }^{\mathrm{e}}$ siècle », in Les études sociales, ${ }^{\circ} 159,2014$, p. 3-9.

${ }^{4}$ G. Poujol, «Éducation populaire : une histoire française », in Hermès, n ${ }^{\circ} 42,2005$, p. 126-130.

${ }^{5}$ L. Mercier, Les universités populaires (1899-1914), Paris, les éd. ouvrières, 1986, p. 177-179.

${ }^{6}$ Les bibliothèques populaires se développent à partir des années 1860 avec des ambitions pédagogiques différentes (A. Boulogne, Des livres pour éduquer les citoyens. Jean Macé et les bibliothèques populaires (18601881), Paris, L'Harmattan, 2016, 213 p.). Les disciples de Durkheim s'emploient à développer des bibliothèques positivistes, alors que la Société Franklin promeut des bibliothèques populaires davantage conformes à la
} 
les cours du soir pour adultes ${ }^{7}$. En rupture avec ces institutions, l'Université populaire prétend offrir des enseignements de qualité universitaire dans les matières traditionnelles (littérature, histoire, philosophie, etc.) sans rechercher une visée professionnelle directement opérante. Ensuite, l'Université populaire se distingue par le public auquel elle se destine : un public adulte et populaire. Ces deux qualificatifs exigent quelques précisions. L'Université populaire s'adresse à un public adulte, c'est-à-dire citoyen. Elle permet ainsi de parfaire l'éducation morale du citoyen exerçant ses droits politiques, notamment celui de suffrage. Enfin, l'Université populaire s'adresse à un public populaire. Là est peut-être sa vocation première, identifiable jusque dans l'expression d' «Université populaire ». Mais ce rapport de l'éducation au peuple nécessite quelques remarques. À quel «peuple» s'adressent les Universités populaires ? Sur ce point, les divergences semblent importantes au sein même du mouvement des «UP ». Le public « populaire » peut désigner un public ouvrier exclu de l'enseignement dispensé pour l'élite. Dans cette perspective, l'Université populaire constitue un lieu d'éducation de l'ouvrier, d'éveil de sa condition matérielle et morale et, dans certaines perspectives, un lieu de préparation de la révolution prolétarienne. Mais, et cela semble avoir été le cas à Lyon, le qualificatif « populaire » peut désigner au sens strict « le peuple », c'est-àdire l'ensemble des citoyens pris sans distinction de leur statut social. Dans cette perspective conforme aux théories de la souveraineté populaire, l'Université populaire a pour objectif de réunir dans un même lieu l'ensemble du corps citoyen divisé. Patrons et ouvriers, riches et pauvres, étudiants et travailleurs doivent pouvoir se réunir autour d'enseignements communs constitutifs d'un socle moral communément partagé dans la République. Cette seconde perspective s'oppose en partie à la première puisqu'elle entend fonder la République sur des bases solides et non préparer une révolution. Cet aspect est particulièrement prégnant lors de la fondation de l'Université populaire lyonnaise et s'explique par le contexte de l'affaire Dreyfus.

Durant la décennie 1890, l'affaire Dreyfus est en effet l'élément déclencheur de la création des Universités populaires. Lors de l'Affaire, la France entière se divise sur le sort réservé au capitaine déchu. Dreyfusards et antidreyfusards s'affrontent sur les questions de société que le scandale judiciaire soulève : l'antisémitisme, la place de l'Église dans l'État, la toute-puissance de l'armée, la persistance de franges anti-démocratiques dans la société, etc. Mais en réalité, l'affrontement entre dreyfusards et antidreyfusards reste cantonné aux salons parisiens, aux salles de conférences et aux prétoires. L'affaire Dreyfus occupe la France des élites qui, au cœur des débats, prennent pour la première fois le qualificatif inventé $\mathrm{d}^{\prime}$ ' intellectuel $»^{8}$. À la Ligue des droits de l'Homme ou à la Ligue de la patrie française, organes symbolisant la lutte intellectuelle accompagnant la lutte judiciaire, on ne trouve guère d'adhérents issus des milieux ouvriers. Au grand désarroi des dreyfusards convaincus que leur combat s'inscrit dans la préservation de la République démocratique et populaire, les ouvriers et paysans se désintéressent de l'actualité ${ }^{9}$. C'est en observant les causes de ce désintéressement que certains dreyfusards entendent poursuivre leur engagement dans une cuvre de

restauration de l'ordre moral (É. Laboulaye, L'éducation du pays par l'armée, Discours à la société Franklin, Paris, Delgrave, 1874, p. 47-72). Édouard Laboulaye est un membre actif de la Société Franklin et partisan de l'éducation populaire (É. Laboulaye, Le parti libéral : son programme et son avenir, 6 éd., Paris, Charpentier, 1863, p. 151158). Voir A. Sandras (dir.), Des bibliothèques populaires à la lecture publique, Villeurbanne, Presses de l'ENSSIB, 2014, 542 p. ainsi que le carnet de recherche en ligne : Histoire des bibliothèques et de la lecture populaires : https://bai.hypotheses.org). À Lyon, les bibliothèques populaires sont créées en 1872 par l'initiative d'Antoine Gailleton, alors conseiller municipal (Archives Municipales de Lyon, 2CM7, p. 330).

${ }^{7}$ Sur les ambiguïtés de l'enseignement à destination de l'ouvrier, voir le n ${ }^{\circ} 159$ des Études sociales (2014) : L'éducation industrielle et les savoirs enseignés aux ouvriers adultes en France (1800-1870).

${ }^{8}$ C. Charle, Naissance des « intellectuels », 1880-1900, Paris, éd. de Minuit, 1990, p. 163-182; J.-F. Sirinelli et P. Ory, Les intellectuels en France, de l'affaire Dreyfus à nos jours, Paris, Perrin, 3éd., p. 19-40 ; M. Winock, Le Siècle des intellectuels, Paris, éd. du Seuil, 1999, p. 11-85.

${ }^{9}$ J.-P. Peter, « Dimensions de l'affaire Dreyfus », in Annales ESC, n 16, 1961, p. 1141-1167. 
rapprochement des élites intellectuelles républicaines et du peuple pris dans son ensemble, en somme de fonder un « usage politique de leur savoir ${ }^{10}$ grâce aux Universités populaires.

À Lyon, ce contexte détermine en 1899 la fondation de l'Université populaire lyonnaise grâce à l'initiative de Jeanne Desparmet-Ruello, directrice de Lycée de jeunes filles ${ }^{11}$. Les séquelles laissées dans la ville par l'Affaire ont rendu nécessaire, pour le camp dreyfusard, la création de cette Université populaire ${ }^{12}$. La composition du groupe fondateur est révélatrice de l'engagement dreyfusard de ses membres. Même si les milieux juridiques lyonnais s'illustrent dans l'antidreyfusisme ${ }^{13}$ et que l'Université de Lyon s'oppose à cette Université populaire ${ }^{14}$, on retrouve tout de même de nombreux juristes parmi les membres qui entourent Jeanne Desparmet-Ruello : Jean Appleton, Louis Josserand, Paul Pic, Justin Godart, Charles Brouilhet et Louis Chazette. D'un point de vue pédagogique, Desparmet-Ruello impose aux conférenciers un esprit général de vulgarisation en rappelant que, malgré le nom d' « Université », la difficulté des cours ne doit pas excéder le niveau du secondaire ${ }^{15}$. Sur le plan matériel, les membres de l'Université populaire lyonnaise font construire une « Maison du peuple » au sein de laquelle les salles de cours côtoient une bibliothèque, un musée, un gymnase, un restaurant ouvrier, des salles de jeux, des salons de lecture et un théâtre ${ }^{16}$. Grâce au prêt d'un bâtiment communal dans le quartier de la Croix-Rousse - rue Hénon -, les locaux de cette Maison du Peuple sont rapidement équipés. L'appui apporté par la municipalité lyonnaise se caractérise par la participation du maire Victor Augagneur à la promotion de $l^{\prime}$ ' $U P{ }{ }^{17}$. La volonté de s'installer dans le quartier ouvrier de la Croix-Rousse est présente dès l'origine du projet car les fondateurs considéraient «qu'au lieu d'appeler à soi la population ouvrière, l'Université devra aller vers elle ${ }^{18}$. Mais, avec le même souci, l’Université populaire lyonnaise tente de rayonner dans les autres quartiers ouvriers de la ville - la Guillotière et Vaise - en créant des « groupes » hébergés dans les locaux de diverses associations ouvrières. Cependant, malgré ces partenariats, l'Université populaire lyonnaise obéit à un projet radical-socialiste qui l'oppose aux œuvres d'éducation populaire influencées par les leplaysiens ou les socialistes ${ }^{19}$.

\footnotetext{
${ }^{10}$ P. Simon-Nahum, « Les intellectuels dreyfusards », in V. Duclert et P. Simon-Nahum (dir.), Les évènements fondateurs. L'affaire Dreyfus, Paris, Armand Colin, 2009, p. 154-157.

${ }^{11}$ L. Roulleau-Berger, «Sociographie des Universités populaires : le cas de Lyon », in Éducation, fête et culture, Lyon, PUL, 1981, p. 105-146.

${ }^{12}$ Édouard Herriot, membre fondateur de l'Université populaire lyonnaise, indique les origines dreyfusardes de 1’UP lyonnaise (Annales de l'Université populaire lyonnaise, $\mathrm{n}^{\circ} 3$, p. 58). Dans ses mémoires, le maire de Lyon revient sur cette époque : «L'affaire Dreyfus provoquait des rapprochements avec les masses ouvrières. Ce fut le temps des universités populaires. Celle dans laquelle je m'enrôlais fonctionnait à la Croix-Rousse, rue Hénon. J'y ai parlé devant des auditoires fort attentifs. J'ai appris, à cette occasion, qu'il ne faut jamais abaisser son enseignement si l'on parle devant le peuple. La culture agit par son ascendant. » (É. Herriot, Jadis, t. 1, Paris, Flammarion, 1948, p. 139).

${ }^{13}$ C. Fillon, Histoire du Barreau de Lyon sous la Troisième République, Lyon, Aléas, 1995, p. 67-88.

${ }^{14}$ Discours de Compayré, Rentrée solennelle de l'Université de Lyon, Lyon, Rey, 1900, p. 49-52. L'Université d'État était hostile à l'usage du mot « université ». Cette opposition a donné lieu à une intervention du préfet : la société initiale - qui prenait le nom de Comité d'initiative pour l'université populaire lyonnaise - a dû changer de nom pour : Société lyonnaise d'instruction et d'éducation populaire (Lyon républicain, 25 octobre 1900).

${ }^{15}$ Annales de l'Université populaire lyonnaise, $\mathrm{n}^{\circ} 2$, p. 7.

${ }^{16}$ Annales de l'Université populaire lyonnaise, $\mathrm{n}^{\circ} 1, \mathrm{p} .3-4$ et $\mathrm{n}^{\circ} 2$, p. 85. Au sein du théâtre, il faut noter la venue des frères Lumière pour des projections avec leur nouveau cinématographe (ibid., p. 55).

${ }^{17}$ Annales de l'Université populaire lyonnaise, $\mathrm{n}^{\circ} 1, \mathrm{p} .13$; $\mathrm{n}^{\circ} 2, \mathrm{p} .19$.

${ }^{18}$ Annales de l'Université populaire lyonnaise, $\mathrm{n}^{\circ} 1$, p. 7.

${ }^{19}$ Les sources utilisées ne témoignent pas d'une volonté manifeste de s'opposer aux autres expériences lyonnaises d'enseignement populaire à destination des adultes. Toutefois, on peut supposer que la démarche des fondateurs de l'Université populaire lyonnaise n'est pas sans rapport avec l'interruption des conférences du vendredi soir tenues aux facultés catholiques lyonnaises (voir dans le présent dossier : M. Biscay, «Les conférences publiques des facultés catholiques de Lyon : l'enseignement d'un droit chrétien, instrument d'une propagande de défense de l'Église »).
} 
En raison de cet attachement politique, l'enseignement du droit devient un enjeu fondamental à l'Université populaire. En effet, alors même que les historiens se sont peu intéressés à cette question, les cours de droit dans les Universités populaires figurent au premier rang des matières approfondies auprès du public profane de la matière. Trois raisons peuvent expliquer l'importance de la place de l'enseignement du droit dans le programme des Universités populaires en général. Premièrement, le projet d'acculturation républicaine des masses ouvrières passe par la nécessité de promouvoir un droit républicain en formation au centre duquel se trouve la réponse juridique à la question sociale ${ }^{20}$. Pour les républicains, enseigner le droit dans les Universités populaires c'est d'abord faire œuvre de propagande en faveur des réformes accomplies et préparer l'opinion aux réformes à venir. Deuxièmement, le contexte de création des Universités populaires - la crise intellectuelle consécutive à l'affaire Dreyfus - influe sur la programmation pédagogique en pensant le droit comme un enjeu éducatif du citoyen. Plus que le droit, il s'agit d'enseigner une certaine conception de la justice et de son rapport à la raison d'État, et donc de porter une critique contre des institutions qui, au mépris de l'évidence, ont condamné Dreyfus. Pour les fondateurs des Universités populaires, la décennie 1890 est une décennie d'interrogation sur le droit et la justice en République. Ces derniers se tournent vers leurs collègues juristes pour repenser le juste en société même si ceuxci peinent à prendre position face aux méandres juridiques de l'affaire Dreyfus ${ }^{21}$. Enfin, troisièmement, il arrive que les juristes soient eux-mêmes impliqués dans les réseaux dreyfusards et politiques qui s'appuient sur les Universités populaires comme lieux de sociabilisation et de promotion sociale. À cet égard, les programmes des Universités populaires sont parfois plus déterminés par la présence de telle ou telle personnalité dans l'équipe fondatrice que par une réflexion aboutie sur l'orientation générale des enseignements.

Dès lors, cette nouvelle formule d'enseignement du droit esquissée à la fin du XIX ${ }^{\mathrm{e}}$ siècle et caractérisée par une pseudo-vulgarisation de la matière juridique s'inscrit en opposition aux formes traditionnelles d'enseignement à l'œuvre au sein des Facultés de droit. Si, tout comme pour l'éducation populaire, il est difficile de démontrer le caractère véritablement populaire d'un tel enseignement, force est de constater qu' au $\mathrm{XIX}^{\mathrm{e}}$ siècle un autre enseignement du droit se développe - moins spécialisé, moins élitiste, plus pratique et plus concret. Mais ce mouvement destiné à mettre «le droit à la portée de tous $»^{22}$ interroge les ambitions des juristes qui l'animent. Il est clair qu'à partir des années 1850, les partisans d'une certaine culture juridique tentent, bien au-delà des débats doctrinaux et judiciaires, de diffuser leurs savoirs au reste de la société avec un souci de vulgarisation ${ }^{23}$. Mais, en reconstituant cette histoire de l'autre enseignement du droit, faisons-nous une histoire populaire du droit? L'expression renvoie immanquablement à l'ouvrage d'Howard Zinn, A People's History of the United States $^{24}$, et aux ouvrages des historiens français qui ont relevé le défi méthodologique d'une

\footnotetext{
${ }^{20}$ A. Stora-Lamarre, La République des faibles. Les origines intellectuelles du droit républicain, 1870-1914, Paris, Armand Colin, 2005, 219 p.

${ }^{21}$ F. Audren et J.-L. Halpérin, La culture juridique française, Paris CNRS éd., 2013, p. 146 et C. Aubisse et P.N. Barenot, «L'ordre ou la justice ? La rébellion manquée des juristes dans l'affaire Dreyfus », in Revue Droit \& littérature, $\mathrm{n}^{\circ} 3,2019$, p. 69-84.

${ }^{22}$ L. Guerlain et N. Hakim (dir.), Littératures populaires du droit. Le droit à la portée de tous, Issy-lesMoulineaux, LGDJ, 2019, 208 p.

${ }^{23}$ L. Guerlain et G. Richard, « Le quotidien et le droit », in Cahiers Jean Moulin, n 5, 2019.

${ }^{24}$ H. Zinn, A People's History of the United States, 1492-present, rééd., New York, Harper Perennial, 1995, 675 p. trad. en français : Une histoire populaire des États-Unis d'Amérique. De 1492 à nos jours, trad. F. Cotton, Marseille, Agone, 2002, 811 p. Pour une nécessaire contextualisation de l'ouvrage : A. Ivol, « "Faire peuple" aux États-Unis : réflexions sur l'histoire populaire d'Howard Zinn », in Revue d'histoire moderne et contemporaine, $\mathrm{n}^{\circ}$ 67-2, 2020, p. 7-26.
} 
histoire populaire ${ }^{25}$. Dans un engouement historiographique, critiquable à certains égards, de plus en plus de disciplines se voient attribuer leur histoire populaire ${ }^{26}$. Mais est-il possible de faire une histoire populaire du droit épistémologiquement conforme au mouvement dit de « l'histoire populaire ${ }^{27}$ ? À cette question, l'historiographie du droit n'a pas encore apporté de réponse méthodologique et, dans le cadre de cette étude, il convient de se préserver de toute volonté de réaliser une histoire populaire de l'enseignement du droit. En effet, les sources qui sont offertes pour comprendre l'enseignement du droit à l'Université populaire lyonnaise, en particulier les publications recueillies dans les Annales de l'Université populaire lyonnaise $(A U P L)^{28}$ limitent la connaissance de cet enseignement au seul point de vue du professeur. Elles condamnent donc toute entreprise de connaissance d'une pratique populaire du droit par le biais de l'étude des Universités populaires. Ainsi, cette étude sur l'enseignement du droit est limitée à l'analyse de l'émission de l'enseignement sans possibilité de traiter de sa réception. Il est nécessaire, par ailleurs, de préciser que les données sur l'appartenance socio-professionnelle des auditeurs n'existent pas. Il faut ajouter qu'être un « non-professionnel » du droit, et donc potentiellement intéressé par un enseignement juridique vulgarisé, ne place pas pour autant un auditeur dans la catégorie du «populaire ». Toutefois, même si la façon dont le droit est enseigné aux Universités populaires ne nous renseigne pas sur la connaissance et la pratique populaires du droit, elle nous permet d'éclairer d'un jour nouveau les stratégies militantes des juristes, professeurs et avocats, qui interviennent dans ces structures d'éducation populaire. L'enseignement du droit devient ainsi un moyen d'intervention dans la vie de la cité, non pas seulement par le contenu des cours, mais par la présence même des juristes enseignants dans les lieux de sociabilité municipale. Derrière des cours qui revêtent une apparente neutralitée ${ }^{29}$, les professeurs de l'Université populaire propagent une certaine conception du droit conforme autant à leurs opinions personnelles qu'au renforcement de leur carrière académique.

Cette conception du droit proposée à un public profane au sein de l'Université populaire lyonnaise interroge. À travers les enseignements dispensés dans la Maison du peuple de la Croix-Rousse, se dessine un projet d'enseignement populaire du droit - ou tout du moins d'un autre enseignement du droit que celui professé à la Faculté de droit. Mais il convient de comprendre comment se caractérise ce projet pédagogique d'un enseignement du droit à destination de tous. À partir du cas de Lyon, on peut tenter d'induire l'identité et la nature de cet enseignement du droit. Deux caractères apparaissent nettement. D'une part, le droit à l'Université populaire est enseigné conformément à une réception du renouveau doctrinal qui s'opère dans la matière juridique à la charnière des $\mathrm{XIX}^{\mathrm{e}}$ et $\mathrm{XX}^{\mathrm{e}}$ siècles (I). D'autre part, le discours sur le droit porté par les enseignants juristes relève d'un discours politique consécutif de l'engagement partisan de ses auteurs (II). Ces conclusions qui déterminent un enseignement $\mathrm{du}$ droit à l'Université populaire fondé sur deux piliers - renouvellement doctrinal et engagement politique - ne sont possibles qu'en appréhendant l'activité d'enseignement au sens

\footnotetext{
${ }^{25}$ En particulier : M. Zancarini-Fournel, Les Luttes et les Rêves. Une histoire populaire de la France de 1685 à nos jours, Paris, La Découverte, 2016, 995 p. et G. Noiriel, Une histoire populaire de la France. De la Guerre de cent ans à nos jours, Marseille, Agone, 2018, 832 p.

${ }^{26}$ É. Ruiz, «L'histoire populaire : label éditorial ou nouvelle forme d'écriture du social ? », in Le mouvement social, $\mathrm{n}^{\circ} 270,2019$, p. 185-230.

${ }^{27}$ Pour un bref aperçu des enjeux épistémologiques de l'histoire populaire : D. Kalifa, « Les historiens français et "le populaire" », in Hermès, $n^{\circ}$ 42, 2005, p. 54-59 et M. Zancarini-Fournel, «Écrire une histoire populaire de la France », in Revue d'histoire moderne et contemporaine, ${ }^{\circ}$ 67-2, 2020, p. 47-62.

${ }^{28}$ À notre connaissance, seuls deux fonds conservent l'intégralité de ces documents à la BNF (8-R-18360) et aux AD du Rhône (PER 1186/1).

${ }^{29}$ Sur ce vœu pieux des juristes : F. Cherfouh, Le juriste entre science et politique. La Revue générale du droit, de la législation et de la jurisprudence en France et à l'étranger (1877-1938), Issy-les-Moulineaux, LGDJ, 2017, p. 237-289 et F. Audren et J.-L. Halpérin, La culture juridique française, op. cit., p. 147.
} 
large, c'est-à-dire en associant l'analyse des cours de droit au contexte dans lesquels ils s'inscrivent.

$\left.\mathrm{I}^{\circ}\right)$ L'enseignement du droit conforme aux exigences sociales, les cours de droit à l'Université populaire comme creuset du renouveau de la recherche juridique

Au tournant du $\mathrm{XX}^{\mathrm{e}}$ siècle à Lyon, le renouvellement de la recherche en droit qui s'opère de façon hétérodoxe au sein de la Faculté se répercute au sein de l'Université populaire ${ }^{30}$. Ce renouvellement se fonde sur la convergence d'une partie du corps professoral autour d'une approche alliant droit et sciences sociales ${ }^{31}$. On observe qu'à l'Université populaire cette approche rénovée de la recherche en droit est réceptionnée et utilisée dans les enseignements, ce qui peut surprendre en raison de l'auditoire profane auquel ceux-ci s'adressent. Mais cela s'explique précisément par l'intérêt porté par les juristes lyonnais aux sciences sociales, ce qui les conduit à participer aux divers groupes d'études de la société qui se forment en dehors de la Faculté. À partir de 1899, l’Université populaire constitue un lieu au sein duquel les juristes côtoient d'autres personnalités issues des sciences sociales et réfléchissent alors à une autre façon d'enseigner le droit. À cet égard, le corps enseignant qui se mobilise au sein de l'Université populaire lyonnaise (A) est significatif de la conjonction qui s'opère dans les enseignements entre droit et sciences sociales (B).

A - Le corps enseignant de l'Université populaire actif dans le renouvellement doctrinal : la promesse d'un enseignement du droit conforme aux exigences sociales

L'enseignement du droit durant les cinq années d'existence de l'Université populaire lyonnaise résulte d'abord d'un phénomène de « médiation locale ${ }^{32}$ entre les juristes lyonnais et un auditoire profane, celui du quartier populaire de la Croix-Rousse. Certains grands noms du renouveau juridique du tournant $d u \mathrm{XX}^{\mathrm{e}}$ siècle donnent des cours dans la Maison du peuple de la rue Hénon avec des degrés d'investissement différents mais avec une présence constante pour tous entre 1899 et 1903.

$\mathrm{Au}$ premier rang des enseignants en droit à l'Université populaire, on trouve deux personnalités qui se distinguent par leur investissement important : Jean Appleton et Louis Chazette. Le premier est professeur à la Faculté de droit de Lyon où il enseigne le droit administratif après un passage par Dijon suite à sa réussite au concours d'agrégation en 1894 . Fils du romaniste Charles Appleton, il est également avocat, poursuivant ainsi une double

\footnotetext{
${ }^{30}$ Sur ce renouvellement de la recherche en droit à la Faculté de Lyon : D. Deroussin (dir.), Le renouvellement des sciences sociales et juridiques sous la Troisième République. La Faculté de droit de Lyon, Paris, La mémoire du droit, 2007, 334 p. et H. Fulchiron (dir.), La Faculté de droit de Lyon, 130 ans d'histoire, Lyon, éd. lyonnaises d'Art et d'Histoire, 2006, 234 p.

${ }^{31} \mathrm{~F}$. Audren, «Comment la science sociale vient aux juristes ? Les professeurs de droit lyonnais et les traditions de la science sociale (1875-1935) », in D. Deroussin (dir.), Le renouvellement des sciences sociales et juridiques, op. cit., p. 3-50. Frédéric Audren insiste toutefois sur les différentes significations que les juristes lyonnais donnent aux sciences sociales : «Ainsi, la revendication collective d'un attachement à la science sociale ne signifie pas pour autant que chacun des auteurs renonce individuellement à des usages et des conceptions spécifiques de celleci : sociologie durkheimienne pour les uns, science sociale leplaysienne pour les autres ou encore sociologie catholique pour les troisièmes (...). L'investissement des juristes lyonnais dans les mondes de la science sociale, la participation à leurs projets et à leurs institutions, contribuent très puissamment à donner une coloration particulière à la production doctrinale de la Faculté et fait rejaillir sur cette dernière l'image d'un établissement à la pointe de l'innovation scientifique que ses membres se plaisent à rappeler et à consolider » (ibid., p. 6).

${ }^{32}$ Ibid.
} 
carrière au sein de la Faculté et du Barreau lyonnais ${ }^{33}$. Dreyfusard convaincu, il s'engage dans tous les lieux de sociabilité qui couvrent l'agglomération rhodanienne à partir de 1898 . Il adhère à la Ligue des droits de l'homme où il rencontre plusieurs des membres fondateurs de l'Université populaire dont Édouard Herriot et le médecin Jean Lépine. Appleton est le type même du juriste engagé qui, suite au scandale de l'affaire Dreyfus, cherche à retourner auprès des citoyens les plus modestes qui ont montré une certaine indifférence face aux péripéties judiciaires ${ }^{34}$. Au sein de l'Université populaire, il est une figure de proue du milieu juridique lyonnais. Il est vice-président du comité d'initiative chargé de penser l'installation de l'Université populaire, puis vice-président de la Société lyonnaise d'instruction et d'éducation populaire, l'association qui supporte l'Université populaire ${ }^{35}$. L'investissement d'Appleton se traduit également par l'appui juridique qu'il apporte pour l'écriture des statuts de l'Université populaire dont il est, avec Chazette, l'auteur ${ }^{36}$. Louis Chazette est l'autre enseignant permanent à l'Université populaire ${ }^{37}$. Il est même celui qui assure le plus d'heures de cours parmi les juristes. Cet avocat, docteur en droit en 1898, futur député élu en 1924 sur la liste radicale menée par Herriot, a commencé sa carrière comme secrétaire de Jean Appleton ${ }^{38}$. Il est probable que ce soit d'ailleurs Appleton qui a mené Chazette vers l'Université populaire ; les deux hommes semblent liés et assurent ensemble la défense lors de grandes affaires ${ }^{39}$. Leur engagement se traduit par la fréquence des cours qu'ils donnent. Chaque semaine, le lundi soir, Chazette ou Appleton enseignent différentes matières juridiques en suivant un programme préétabli. Même si la présence d'Appleton et de Chazette n'est pas en elle-même représentative du renouvellement doctrinal, il faut l'interpréter comme une interface entre l'Université populaire et d'autres enseignants de la Faculté de droit à la présence plus ponctuelle.

En effet, à côté des deux enseignants permanents que sont Appleton et Chazette, plusieurs professeurs donnent des cours à l'Université populaire de façon ponctuelle, à raison d'une ou deux fois par an. Ces enseignants juristes plus occasionnels sont, eux, révélateurs de la réception au sein de l'Université populaire lyonnaise du renouvellement de la doctrine qui s'opère en même temps à la Faculté de droit. Parmi ces enseignants à l'Université populaire, on note la présence dès le début de Louis Josserand ${ }^{40}$, de Paul Pic ${ }^{41}$ et de Justin Godart ${ }^{42}$. À partir de 1903, ceux-ci sont rejoints par deux de leurs collègues de la Faculté et du Barreau : Emmanuel Lévy ${ }^{43}$ et Marius Moutet ${ }^{44}$, témoignant ainsi d'une présence socialiste plus tardive au sein de l'Université populaire. La présence de ces professeurs qui donnent environ deux soirées de cours par an est révélatrice de la réception des orientations doctrinales au sein de l'Université populaire. Chacun de ces enseignants participent, dans son domaine, au renouvellement de la recherche en droit. Paul Pic et Justin Godart animent dans les mêmes

\footnotetext{
${ }^{33}$ Sur Jean Appleton : C. Fillon, «L'itinéraire d'un avocat engagé, l'exemple de Jean Appleton (1868-1942)», Revue internationale d'histoire de la profession d'avocat, $\mathrm{n}^{\circ}$ 6, 1994, p. 195-218 et Histoire du Barreau de Lyon, op. cit., p. 72-86.

${ }^{34}$ Ibid., p. 75.

${ }^{35} A U P L, \mathrm{n}^{\circ} 1$, p. 9 et 16.

${ }^{36} A U P L, \mathrm{n}^{\circ} 1$, p. $12 ; \mathrm{n}^{\circ} 4$, p. 90.

${ }^{37}$ Chazette demeure une personnalité peu connue (C. Fillon, Histoire du Barreau de Lyon, op. cit., p. 277-279).

${ }^{38}$ Voir la correspondance entre Chazette et Josserand : AD Rhône, $224 \mathrm{~J} 27$.

${ }^{39}$ Les deux avocats défendent ensemble pour le compte de la Ligue des droits de l'homme Kissel et Hildesheimer victimes d'attaques antisémites en 1899 par le journal l'Antijuif lyonnais et stéphanois et Mlle Machillot, une serveuse arrêtée et internée sans jugement par la police des mœurs en 1902.

${ }^{40} A U P L, \mathrm{n}^{\circ} 3$, p. $1, \mathrm{n}^{\circ} 4$, p. 3.

${ }^{41} A U P L, \mathrm{n}^{\circ} 1$, p. $31 ; \mathrm{n}^{\circ} 4$, p. 3.

${ }^{42} A U P L, \mathrm{n}^{\circ} 4$, p. $3 ; \mathrm{n}^{\circ} 4$, p. $75 ; \mathrm{n}^{\circ} 5$, p. 4 et 100.

${ }^{43}$ AUPL, $\mathrm{n}^{\circ}$ 5, p. 4, 49 et 83 .

${ }^{44}$ Ibid., p. 100 et 110.
} 
années la revue Questions pratiques de législation ouvrière et d'économie sociale ${ }^{45}$ qui est conçue comme un dépassement d'une simple approche théorique du droit social pour tendre vers une alliance entre recherche en droit et recherche en économie sociale ${ }^{46}$. Emmanuel Lévy, principal représentant du courant du socialisme juridique ${ }^{47}$, Marius Moutet, avocat, tout comme Louis Josserand ${ }^{48}$, proposent quant à eux une approche rénovée de leurs champs disciplinaires. Ces juristes à l'influence nationale se retrouvent autour d'un dénominateur commun qui est l'usage des sciences sociales en droit. À cet égard, il est d'ailleurs significatif qu'à partir de 1902 la catégorie qui regroupe les enseignements juridiques dans les programmes de l'Université populaire lyonnaise ne s'intitule plus seulement «droit » mais « droit et sciences sociales $»^{49}$.

Pour compléter ce panel d'enseignants, il faut relever la présence, chaque année, de personnalités invitées par l'Université populaire à donner des conférences inscrites dans le programme des cours. Parmi ces conférences, certaines traitent directement de questions juridiques, d'autres abordent l'étude du droit par le biais d'autres disciplines. Paul Huvelin, historien du droit à la Faculté de Lyon, est ainsi invité à donner une leçon en 1903 sur «la famille à travers les âges ${ }^{50}$, titre qui laisse penser à une conférence grand public sur l'histoire du droit prise à travers un prisme anthropologique. Comme la plupart des juristes intervenant à l’Université populaire, Huvelin a marqué le renouvellement de la recherche en droit au tournant $\mathrm{du} \mathrm{XX}^{\mathrm{e}}$ siècle en usant, lui aussi, des sciences sociales, en particulier de l'anthropologie, par un attachement aux enseignements de Durkheim ${ }^{51}$. À côté d'Huvelin, d'autres conférenciers invités traitent de façon large de questions juridiques - ou plus spécifiquement judiciaires. C'est le cas notamment durant l'année 1901 avec la venue d'Alexandre Lacassagne, alors professeur à la Faculté de médecine, pour aborder une question de criminologie : «le tatouage $»^{52}$. La

\footnotetext{
${ }^{45}$ Dans l'esprit de l'enseignement du droit à destination de tous, ils participent également à la Revue populaire d'économie sociale pour la vulgarisation des sciences économiques et des æuvres sociales (1902, p. 168 ; 1903 , p. 107).

${ }^{46}$ N. Hakim, «Une revue lyonnaise au cœur de la réflexion sur le droit social : les Questions pratiques de législation ouvrière et d'économie sociale », in Le renouvellement des sciences sociales et juridiques, op. cit., p. 123-152. Sur Pic : D. Bayon et L. Frobert, «Paul Pic (1862-1944) et les "lois ouvrières" », in Revue d'histoire des Facultés de droit et de la science juridique, $\mathrm{n}^{\circ}$ 18, 1997, p. 69-94 ; « Lois ouvrières et réformisme social dans l'œuvre de Paul Pic (1862-1944) », in Le mouvement social, n 201, 2002, p. 53-80 ; N. Dockès-Lallement, «Paul Pic », in La Faculté de droit de Lyon, 130 ans d'Histoire, op. cit., p. 103-107; N. Hakim, «La science de la question sociale de Paul Pic », in N. Hakim et F. Melleray (dir.), Le renouveau de la doctrine française, Paris, Dalloz, 2009, p. 123-158 ; F. Lekéal, «Paul Pic entre Code civil et Code du travail : les voies du réformisme juridique », in Le renouvellement des sciences sociales et juridiques, op. cit., p. 153-171. Les conceptions de Justin Godart, chargé de cours à la Faculté de droit, demeurent encore peu connues en comparaison avec les travaux réalisés sur son parcours de député et de ministre (A. Wieviorka (dir.), Justin Godart. Un homme dans son siècle (1871-1956), Paris, CNRS éd., 2004, 261 p.).

${ }^{47}$ Sur le socialisme juridique de Lévy et son rapport aux sciences sociales, voir le numéro 57 de la revue Droit et société (2004); C. M. Herrera, «Socialisme juridique et droit naturel. À propos d'Emmanuel Lévy », in C. M. Herrera (dir.), Les juristes face au politique, Paris, Kimé, 2003, p. $69-84$; « Droit et socialisme à la Faculté de droit de Lyon», in Le renouvellement des sciences sociales et juridiques, op. cit., p. 279-302; «La radicalisation sociologique de la pensée juridique. Lévy, Lambert, Gurvitch », in Droits, n 62, 2015, p. 189-204. ${ }^{48}$ Sur les rapports novateurs que Josserand établit entre la science juridique et la science sociale : D. Deroussin, «L. Josserand : le droit comme science sociale ? », in Le renouvellement des sciences sociales et juridiques, op. cit., p. 63-119; C. Fillon et F. Audren, «Louis Josserand ou la construction d'une autorité doctrinale », in RTDciv, $\mathrm{n}^{\circ} 1,2009$, p. 39-76.

${ }^{49} A U P L, \mathrm{n}^{\circ} 5, \mathrm{p} .4$.

${ }^{50}$ Ibid., p. 49.

${ }^{51}$ F. Audren, « Paul Huvelin (1873-1924) : juriste et durkheimien », in Revue d'histoire des sciences humaines, $\mathrm{n}^{\circ} 4,2001$, p. 117-130. Huvelin semble n'être intervenu qu'à une seule reprise, et tardivement, à l'Université populaire, ce qui amène à nuancer l'idée qu'il « participe activement à l'Université populaire lyonnaise » (Ibid., p. 124).

${ }_{52}$ AUPL, $\mathrm{n}^{\circ} 3$, p. 47-48. La conférence reprend, pour l'étude des criminels, les conclusions de Lacassagne exprimées dès 1881 : A. Lacassagne, Les tatouages : étude anthropologique et médico-légale, Paris, Baillière,
} 
même perspective médico-légale appliquée au droit est reprise pour une autre conférence par un de ses élèves, membre de l'Université populaire, Georges Beauvisage, qui accorde une conférence sur 《l'état civil et l'acte de mariage ${ }^{53}$. Dans une perspective plus internationaliste, 1'Université populaire lyonnaise reçoit également Charles Richet, intervenant sur « la paix $»^{54}$, et Frédéric Passy, intervenant sur «la guerre et la paix ${ }^{55}$. Dans les petits locaux de la rue Hénon, ces deux conférences visent à promouvoir le recours à l'arbitrage comme mode de résolution des conflits entre États. Richet et Passy profitent du climat intellectuel consécutif de l'affaire Dreyfus pour diffuser les idées portées par la Société française de l'arbitrage entre nations alors gagnée par une approche sociologique des conflits armées ${ }^{56}$. Après la réception de ces deux personnalités à la stature internationale, l'Université populaire lyonnaise accueille en 1903 pour une soirée sur la justice et la laïcité consacrée au centenaire de la naissance d'Edgar Quinet, le colonel Picquart, héros pour un temps du camps dreyfusard ${ }^{57}$. Enfin, il faut noter la présence curieuse de Georges Vallet, docteur en droit, magistrat à Saint-Étienne et auteur de manuels de «droit usuel ${ }^{58}$, pour une conférence d'archéologie sur les aqueducs romains ${ }^{59}$.

Ainsi, qu'ils soient permanents, ponctuels ou invités, les enseignants à l'Université populaire lyonnaise indiquent clairement une orientation pédagogique certaine : le droit, même à destination des profanes, relève d'un rapport étroit avec les sciences sociales. Cette orientation s'observe dans le contenu même des enseignements qui portent, devant ce public profane, une vision du droit associé aux sciences sociales.

1881, 115 p. Sur l'usage des sciences sociales par Lacassagne : H. Souchon, « Alexandre Lacassagne et l'École de Lyon. Réflexions sur les aphorismes et le concept de "milieu social" », in Revue de science criminelle et de droit pénal comparé, $\mathrm{n}^{\circ} 3,1974$, p. 533-559.

${ }^{53} A U P L, \mathrm{n}^{\circ} 1$, p. 10. Derrière l'intitulé juridique se dissimule une conférence reprenant la critique des articles du Code civil relatifs à l'état civil formulée par Lacassagne (A. Lacassagne, Les actes de l'état civil. Étude médicolégale de la naissance, du mariage et de la mort, Bibliothèque scientifique judiciaire de l'avocat et du magistrat, Lyon, Storck, 1887, 223 p.).

${ }^{54} A U P L, \mathrm{n}^{\circ} 4$, p. 53 et $65-72$.

${ }^{55} A U P L, \mathrm{n}^{\circ} 5$, p. 26.

${ }^{56}$ Sur leur approche de la guerre, de la paix et de l'arbitrage par les sciences sociales, voir notamment : C Richet, «L'idée de l'arbitrage international est-elle une chimère ? ", in Revue internationale de sociologie, $\mathrm{n}^{\circ}$ 5, 1896, p. 376-382 et F. Passy, «L'économie politique et la paix », in La paix et l'enseignement pacifique : leçons professées à l'École des hautes études sociales, Paris, Félix Alcan, 1904, p. 9-24. L'approche de l'arbitrage par les sciences sociales sera continuée dans les milieux radicaux lyonnais de l'entre-deux-guerres. Membres de l'Université populaire lyonnaise, Édouard Herriot et Justin Godart sont les principaux partisans d'une approche solidariste de l'arbitrage international à la Société des Nations (J. Henning, Le radicalisme d'Édouard Herriot et la crise des institutions, Paris, Dalloz, 2019, p. 93-94).

${ }^{57}$ AUPL, $\mathrm{n}^{\circ}$ 5, p. 49.

${ }^{58}$ Georges Vallet est aussi un auteur de manuels de droit pour les étudiants (Tableaux synoptiques, contenant l'histoire du droit romain et les concordances avec l'histoire politique des origines à Justinien, conformes à l'esprit des nouveaux programmes, Paris, Larose, 1897, 27 p.), pour les magistrats (Manuel des magistrats du parquet et des officiers de police judiciaire, suivi d'un code de citation et d'audience, et renfermant toutes les formules usuelles, 2 t., Paris, Larose, 1890, 669 et 650 p.) et à l'égard du grand public (Dictionnaire-manuel de droit usuel, Paris, Recueil des lois usuelles, 1896, 680 p.). Sur les formes de « littératures populaires du droit »: L. Guerlain et N. Hakim (dir.), Littératures populaires du droit, op. cit.

59 À l'occasion d'une balade-conférence, Vallet s'autorise quelques considérations politiques : « Admirons sans réserve les œuvres d'autrefois, mais n'oublions pas non plus que des travaux de cette nature n'étaient possibles que dans un État où ne se posait pas la question sociale, résolue alors par l'esclavage » $\left(A U P L, \mathrm{n}^{\circ} 3\right.$ bis, p. 18). 
B - Des cours conformes au renouveau de la science juridique : une réponse aux objectifs pratiques de l'enseignement du droit

Le choix des matières enseignées à l'Université populaire témoigne de la vision des partisans d'un enseignement du droit vulgarisé. À la Maison du peuple de la Croix-Rousse, on ne trouve pas les enseignements classiques qui ont lieu, à la même époque, dans les Facultés de droit d'État. Le découpage traditionnel des cours qui faisait la part belle au droit civil et au droit romain - bien qu'atténué suite aux décrets du 24 juillet 1889 et du 30 avril 1895 accordant plus de diversité dans les enseignements juridiques ${ }^{60}-$, n'a pas lieu à l'Université populaire qui s'affranchit des traditions pédagogiques du $\mathrm{XIX}^{\mathrm{e}}$ siècle pour un enseignement à la fois utile au citoyen et fidèle au renouvellement de la recherche juridique.

Afin de répondre à ces exigences, il faut noter la primauté des cours de droit social, ou plutôt, pour reprendre l'intitulé exact employé au début du XX $\mathrm{X}^{\mathrm{e}}$ siècle, des cours de "législation ouvrière ${ }^{61}$. Pour la première année de cours, les juristes s'accordent sur l'idée que l'enseignement régulier du lundi soir doit être consacré à la législation ouvrière, cours qui recouvre celui de législation industrielle de la Faculté de droit ${ }^{62}$. Ce choix ne semble pas avoir fait l'objet de discussions, ce qui montre que la matière est alors considérée comme incontournable pour le public profane auquel elle s'adresse. L'année suivante, Chazette refait le même cours mais en l'exportant dans le quartier Perrache en étant hébergé par le groupe d'études sociales du $2^{\mathrm{e}}$ arrondissement ${ }^{63}$. Proche des préoccupations pratiques des ouvriers et fer de lance du mouvement de rénovation doctrinale à l'œuvre à la Faculté de Lyon, ce cours de législation ouvrière dispose d'un volume d'environ 22 heures réparties en 11 séances de deux heures ${ }^{64}$. C'est Louis Chazette qui se charge presque exclusivement du cours avec l'aide ponctuelle de Paul Pic pour la conférence d'ouverture ${ }^{65}$. Les Annales de l'Université populaire permettent de connaître le programme de ce cours et montrent qu'il ne s'agit pas seulement d'un exposé de la législation en vigueur :

«Contrat de louage des services ; Syndicats professionnels; Sociétés coopératives de production, de consommation, de construction et de crédit ; Privilèges relatifs aux salaires ; Insaisissabilité, modes et époques de paiement des salaires ; Apprentissage ; Réglementation du travail des enfants, des femmes et des adultes ; Accidents de fabrique, responsabilité, risques professionnels, sociétés industrielles pour la prévention des accidents; Lois ou questions législatives concernant les institutions de prévoyance et d'épargne (caisse des retraites pour la vieillesse, caisses d'assurances, sociétés de secours mutuels). » ${ }^{66}$

Le choix des thèmes enseignés montre le souhait de l'Université populaire de proposer un enseignement pratique directement mobilisable par les auditeurs. Cette ambition d'une vulgarisation - et donc d'une réception - du droit social semble être au cœur du projet d'enseignement juridique à l'Université populaire ${ }^{67}$. Elle rencontre l'un des défis de cette

\footnotetext{
${ }^{60}$ F. Audren et J.-L. Halpérin, op. cit., p. 119.

${ }^{61} A U P L, \mathrm{n}^{\circ} 2$, p. 31.

${ }^{62}$ F. Lekéal, «Les origines de l'enseignement de la législation industrielle : enjeux et qualification d'un nouveau champ d'études », in F. Hordern (dir.), Construction d'une Histoire du droit du travail, Cahiers de l'Institut Régional du Travail, $\mathrm{n}^{\circ}$ 9, 2001, p. 21-41.

${ }^{63}$ AUPL, $\mathrm{n}^{\circ} 4$, p. 38-43.

${ }^{64} A U P L, \mathrm{n}^{\circ} 2$, p. 54-64.

${ }^{65}$ Ibid., p. 31.

${ }^{66}$ Ibid., p. 50. Dans un «aperçu des idées générales développées dans le cours », Chazette détaille son enseignement avec des intitulés plus engagés encore donnant une idée de la tonalité du cours : «Les ennemis de la coopération », « Recherche des causes de la situation précaire des salaires », « De la théorie du salaire minimum », «Thèse de la Révolution sociale. Thèse de l'évolution » (ibid., p. 56).

${ }^{67}$ Cette démarche est, au même moment, également au cœur de la fondation de la revue Questions pratiques de législation ouvrière ( $\mathrm{N}$. Hakim, «Une revue lyonnaise au cœur de la réflexion sur le droit social : les Questions pratiques de législation ouvrière et d'économie sociale », op. cit., p. 135-141).
} 
branche du droit : réduire la technicité pour être mobilisable par le plus grand nombre ${ }^{68}$. Ainsi, les approches théoriques sont a priori écartées du cours au sens strict mais conservent une place dans des cours secondaires dispensés immédiatement après les cours de législation ouvrière. En effet, Chazette a fait préciser dans les programmes que « des cours de sociologie, ou de morale sociale pourront être faits après le cours de droit ${ }^{69}$. Le contenu de ces cours de sociologie associés au cours de législation ouvrière demeure inconnu mais il montre l'alliage tenté, à l'Université populaire, entre considération pratique des enseignements et incorporation du renouvellement de la recherche en droit. L'ombre de Paul Pic est d'ailleurs partout dans cet enseignement. Plusieurs intitulés de thèmes renvoient directement à des sections du Traité élémentaire de législation industrielle paru pour la première fois en $1894^{70}$. Paul Pic ouvre luimême le cours de Chazette par une conférence inaugurale intitulée « La législation ouvrière et la démocratie », dont la transcription complète a été réalisée dans les Annales ${ }^{71}$. La conférence permet d'abord à Pic d'accorder sa confiance à Chazette, montrant l'influence du maître du droit du travail sur l'Université populaire ${ }^{72}$. Ensuite, la conférence sert surtout pour Paul Pic à développer une approche nouvelle des questions juridiques relatives au travail. $\mathrm{Si}$ sa préoccupation est d' "indiquer l'orientation que [la législation ouvrière] doit, à mon avis, recevoir dans une démocratie comme la nôtre $»^{73}$, Paul Pic fait une application de la théorie des milieux en distinguant l'évolution du droit du travail dans un «milieu monarchique » et dans un «milieu démocratique $»^{74}$. Sa conférence témoigne d'une méthode juridique nouvelle en accordant de larges développements historiques, comparés, et appliqués par des études au niveau local - en l'occurrence lyonnais. Outre cette conférence fondatrice, Paul Pic intervient parfois en complément du cours de Louis Chazette, notamment pour promouvoir l'usage de l'économie sociale dans l'étude du droit ${ }^{75}$. Cette approche de l'enseignement du droit par l'économie sociale se retrouve également dans les enseignements dispensés par le principal collaborateur de Paul Pic: Justin Godart. À partir de 1901, celui-ci enseigne un cours d' ' économie politique et sociale ${ }^{76}$. Ainsi, devant l'auditoire de la Croix-Rousse, il accorde plusieurs heures à différents thèmes : «Les tisseurs lyonnais à travers les âges », «Le familistère de Guise », "Histoire de l'évolution du travail (de l'esclavage à la coopération) », «Le mouvement coopératif à Lyon ${ }^{77}$. D'autres personnalités dispensent quelques cours et conférences à l'Université populaire, montrant ainsi la permanence d'un fond de droit social parmi les enseignements juridiques. On note ainsi des cours sur «les coopératives de consommation » $(\mathrm{M} \text {. Ménard })^{78}$ ou sur «les idées religieuses et l'assistance sociale » (M. Moutet $)^{79}$.

À côté de cette primauté accordée au droit du travail dans les enseignements juridiques à l'Université populaire, on note également l'importance des cours de droit public. C'est ainsi qu'après une année d'enseignement du droit social, Louis Chazette et Jean Appleton proposent

\footnotetext{
${ }^{68}$ A.-S. Chambost et A. Mages, La réception du droit du travail par les milieux professionnels et intellectuels, Issy-les-Moulineaux, LGDJ, 2017, p. 8-13.

${ }^{69} A U P L, \mathrm{n}^{\circ} 2$, p. 50.

${ }^{70}$ La table des matières du Traité renvoie à certains intitulés identiques ou proches comme : « Réglementation du travail industriel des femmes et des enfants ; Réglementation du travail des hommes adultes » (P. Pic, Traité élémentaire de législation industrielle, Paris, Rousseau, 1894, p. 625-629).

${ }^{71}$ AUPL, $\mathrm{n}^{\circ} 2$, p. 31-49.

72 À l'auditoire, Paul Pic indiquait ainsi : «Je me garderais bien d'entrer aujourd'hui dans le détail de cette législation, car mon ancien élève et ami, M. Chazette, s'acquittera à merveille de cette tâche » (ibid., p. 31).

${ }^{73}$ Ibid.

${ }^{74}$ Ibid., p. 36-37.

${ }^{75}$ En 1902, il dispense un cours sur « Les assurances ouvrières et la mutualité libre » $\left(A U P L, \mathrm{n}^{\circ} 4\right.$, p. 3$)$.

${ }^{76}$ Ibid.

${ }^{77}$ AUPL, ${ }^{\circ} 4$, p. $75, \mathrm{n}^{\circ} 5$, p. 4, 100.

${ }^{78} A U P L, \mathrm{n}^{\circ} 3$, p. 34.

${ }^{79} A U P L, \mathrm{n}^{\circ}$ 5, p. 100.
} 
à la Maison du peuple un enseignement de droit constitutionnel. Durant l'année scolaire 19011902, les deux hommes se partagent le programme: Chazette s'occupe du «droit constitutionnel » au sens strict, c'est-à-dire des questions institutionnelles, et Appleton des «libertés publiques ${ }^{80}$. Les deux enseignements sont complémentaires mais ils semblent aussi répondre à une volonté d'introduire à l'Université populaire la subdivision du droit public entre droit constitutionnel et droit administratif. En effet, le cours de libertés publiques d'Appleton doit être compris comme un cours rattaché aux logiques du droit administratif conformément à la répartition disciplinaire en usage au $\mathrm{XIX}^{\mathrm{e}}$ siècle $^{81}$. Si l'enseignement du droit constitutionnel est alors récent dans les Facultés de droit ${ }^{82}$, il occupe une place importante à l'Université populaire. Chazette, fidèle à sa méthode d'enseignement, établit un programme clair qui témoigne là encore d'un alliage entre considérations pratiques - électorales - et réflexions novatrices. Ses cours traitent ainsi, dans un ordre significatif d'une prise de recul par rapport aux lois de 1875 , des thèmes suivants :

Étude de la Constitution de 1875 ; Les pouvoirs publics. Le Président de la République ; Attributions du Président de la République ; La Chambre des Députés, ses attributions ; Attributions des ministres et leur responsabilité; Attributions du Sénat; Des divers modes d'élection; La représentation proportionnelle avec un essai pratique de scrutin $»^{83}$.

Dans une certaine mesure, ce plan de cours reprend le plan de la deuxième partie du manuel d'Adhémar Esmein, Éléments de droit constitutionnel français et comparé ${ }^{84}$, dont la seconde édition, en 1899, connait un fort succès éditorial et s'impose comme le « livre officiel des révisions du cours semestriel d'éléments de droit constitutionnel ${ }^{85}$. Sans lecture linéaire des textes de 1875, le cours de Chazette reflète une vision républicaine modérée des institutions en excluant tout débat sur le révisionnisme. Si Chazette dispense un cours général, Jean Appleton se contente plutôt d'un cours thématique en accordant plusieurs heures au même thème «Les libertés publiques et la liberté de conscience », sans que l'on sache exactement le contenu de ce cours, faute de sources. L'année suivante (1902-1903), Jean Appleton propose un nouveau cours de libertés publiques intitulé «Commentaire de la Déclaration des droits de $l^{\prime}$ homme et du citoyen » en dix leçons de deux heures ${ }^{86}$. Là encore, les sources dont nous disposons ne nous permettent pas de connaître le contenu de ces leçons, mais il est probable qu'Appleton ait choisi ce thème en connaissant la controverse juridique qui a lieu durant les mêmes années entre Jellinek et Boutmy sur les origines de la Déclaration ${ }^{87}$. Ces enseignements de droit public répondent sans dissimulation à une volonté d'apporter, dans l'ensemble des disciplines, une éducation politique aux citoyens. Dans un rapport annuel, Édouard Herriot, secrétaire de l'Université populaire, ne s'en cache pas en expliquant la philosophie qui gouverne les enseignements :

«Il se trouvait, en effet, que depuis trente ans de République, certains problèmes avaient été abordés, mais dans l'ensemble, un problème restait intact malgré l'admirable extension de l'enseignement

\footnotetext{
${ }^{80} A U P L, \mathrm{n}^{\circ} 4$, p. 3-5.

${ }^{81}$ G. Richard, Enseigner le droit public à Paris sous la Troisième République, Paris, Dalloz, 2015, p. 300. Il faut rappeler que Jean Appleton est lui-même chargé du cours de droit administratif à la Faculté de Lyon.

82 Ibid., p. 48 et $137-150$.

${ }^{83}$ AUPL, n ${ }^{\circ} 4$, p. 12, 15, 43, 53.

${ }^{84}$ A. Esmein, Éléments de droit constitutionnel français et comparé, 2e éd., Paris, Larose, 1899, 794 p.

${ }^{85}$ G. Sacriste, «Droit, Histoire et politique. Sur quelques implications politiques de la méthode du droit constitutionnel à la fin du XIX ${ }^{\mathrm{e}}$ siècle », in Revue d'histoire des sciences humaines, ${ }^{\circ}$ 4, 2001, p. 87.

${ }^{86} A U P L, \mathrm{n}^{\circ} 5$, p. 4.

${ }^{87}$ Dans l'ordre des répliques : G. Jellinek, La Déclaration des droits de l'Homme et du Citoyen, contribution à l'histoire du droit constitutionnel moderne, trad. G. Fardis, Paris, A. Fontemoing, 1902, p. 93-101 ; É. Boutmy, «La Déclaration des droits de l'Homme et du Citoyen et M. Jellinek », in Annales des sciences politiques, t. 17, 1902, p. 415-441; G. Jellinek, « La Déclaration des droits de l'homme et du citoyen », Revue de droit public et de sciences politiques, 1902, p. 385-400 (D. Klippel, «La polémique entre Jellinek et Boutmy : une controverse scientifique ou un conflit de nationalismes », in Revue française d'histoire des idées politiques, $\mathrm{n}^{\circ} 1,1995$, p. 7994).
} 
primaire, malgré la bonne volonté de l'enseignement secondaire, malgré l'éminente dignité de l'enseignement supérieur et ce problème c'était pour nous le problème essentiel, le problème vital, puisque c'était le problème de l'éducation de la démocratie ${ }^{88}$.

À côté de ces deux piliers de l'enseignement du droit à l'Université populaire que sont le droit social et le droit public, on observe un certain nombre d'enseignements sur d'autres matières juridiques. Il faut noter d'abord le cours curieusement prospectif bien que vulgarisé de Chazette durant l'année scolaire 1902-1903 sur «Droit privé et droit public ${ }^{89}$. En pas moins de seize soirées réparties durant l'année, Louis Chazette s'attaque devant un auditoire profane à la problématique difficile de la délimitation des champs juridiques du droit privé et du droit public. En discutant à chaque cours d'une catégorie - comme « le mariage », « la famille », « le domicile», «les funérailles ${ }^{90}$ - Chazette semble tenter de déterminer les critères de distinction de la traditionnelle summa divisio démontrant par là un souci d'intégrer au sein de l'Université populaire les débats qui animent alors les juristes ${ }^{91}$. Ensuite, il faut souligner le souci de dispenser quelques enseignements relatifs au droit fiscal, tels que deux cours donnés en 1900 par Appleton portant l'un sur « la justice dans l'impôt » et l'autre sur « la question de l'octroi ». Le premier cours, hébergé par le cercle populaire de Vaise, est l'occasion pour le professeur de droit administratif de réfuter les thèses classiques sur la proportionnalité de l'impôt ${ }^{92}$. Le second vise à préciser l'intérêt de penser une imposition indirecte orientée vers des objectifs sanitaires et sociaux ${ }^{93}$. C'est dans ce même esprit de justice que plusieurs cours sur l'institution judiciaire au sens large sont également organisés. Ces cours sont directement liés à une analyse critique des institutions judiciaires dans le contexte de l'affaire Dreyfus. Jean Appleton accorde ainsi une conférence sur « la jurisprudence du président Magnaud ${ }^{94}$. Cette conférence sur le «bon juge Magnaud » ${ }^{95}$ qui a consacré l'état de nécessité se comprend par rapport à la défiance des républicains à l'égard d'une magistrature considérée comme conservatrice. Elle permet aux dreyfusards de l'Université populaire de construire l'idéal d'une magistrature républicaine débarrassée de ses oripeaux réactionnaires ${ }^{96}$. En mars 1903, ce même questionnement sur le juge structure une conférence d'Emmanuel Lévy - «le juge et la loi »dont on ignore le contenu mais dont on suppose la tonalité critique ${ }^{97}$. Enfin, il faut remarquer le choix fait à partir de l'année 1902 d'apporter à l'Université populaire quelques enseignements historiques du droit, notamment un nouveau cours de Chazette en plusieurs

\footnotetext{
${ }^{88} A U P L, \mathrm{n}^{\circ} 3$, p. 60.

${ }^{89} A U P L, \mathrm{n}^{\circ} 5, \mathrm{p} .4$.

${ }^{90}$ Ibid., p. 49.

${ }^{91}$ Les débats sur la détermination de la summa divisio connaissent une actualité particulière au tournant du $\mathrm{XX}^{\mathrm{e}}$ siècle en réceptionnant le renouvellement doctrinal à l'œuvre en France (L.-A. Barrière, «Une approche historique de la summa divisio droit public-droit privé », in B. Bonnet et P. Deumier (dir.), De l'intérêt de la summa divisio droit public-droit privé, Paris, Dalloz, 2010, p. 7-30 ; G. Bigot, « La distinction du droit public et du droit privé au prisme de la doctrine française du premier XIX ${ }^{\mathrm{e}}$ siècle », in P. Alvazzi del Frate, S. Bloquet et A. Vergne (dir.), La summa divisio droit public / droit privé dans l'histoire des systèmes juridiques en Europe (XIX ${ }^{e}-X_{X I}^{e}$ siècles), Bayonne, Institut Universitaire Varenne, 2018, p. 15-36 ; B. Barraud, «Droit public - droit privé : de la summa divisio à la ratio divisio ?», in Revue de la recherche juridique. Droit prospectif, 2014, p. 1101-1132).

${ }^{92}$ AUPL, $\mathrm{n}^{\circ} 2$, p. 12.

${ }^{93}$ Ibid., p. 59.

${ }^{94}$ Ibid., p. 66. La conférence suit la publication en 1900 d'une anthologie des jugements rendus par Paul Magnaud (H. Leyret, Les jugements du Président Magnaud, Paris, Stock, 1900). Sur la portée juridique de l'affaire de la veuve Ménard : M.-A. Frison-Roche, «Le modèle du bon juge Magnaud », in De code en code. Mélanges en l'honneur de Georges Wiederkehr, Paris, Dalloz, 2009, p. 335-342 ; D. Veillon, « Les juristes et le vol de nécessité au tournant des $\mathrm{XIX}^{\mathrm{e}}$ et $\mathrm{XX}^{\mathrm{e}}$ siècles », in F. Chauvaud et A.-D. Houte (dir.), Au voleur! Images et représentations $d u$ vol dans la France contemporaine, Paris, Publications de la Sorbonne, 2014, p. 175-185.

${ }^{95}$ L'expression est utilisée par Clemenceau (L'Aurore, 14 mars 1898).

${ }^{96}$ A.-D. Houte, « Le bon juge Magnaud et l'imaginaire de la magistrature à l'aube du XX $\mathrm{XX}^{\mathrm{e}}$ siècle », in Délibérée, $\mathrm{n}^{\circ} 5,2018$, p. 38-42.

${ }^{97} A U P L, \mathrm{n}^{\circ} 5$, p. 83 .
} 
leçons intitulé «l'évolution de la propriété à travers les âges ${ }^{98}$. Ce cours semble avoir été pensé en synergie avec la conférence donnée par Paul Huvelin sur «la famille à travers les âges ${ }^{99}$ et les cours donnés par Justin Godart sur «l'histoire de l'évolution du travail (de l'esclavage à la coopération) ${ }^{100}$.

Dans ces enseignements juridiques, il faut remarquer la place très faible accordée au droit civil. En effet, à part deux conférences, l'une de Josserand sur « la situation légale de la femme $»^{101}$ et l'autre de Lévy sur «la propriété ${ }^{102}$, on observe le très faible intérêt des organisateurs pour les matières qui sont, pourtant, au tournant $\mathrm{du} \mathrm{XX}^{\mathrm{e}}$ siècle, au cœur des enseignements dans les Facultés de droit.

À travers ces cours, se dessine une certaine conception de l'enseignement du droit à l'Université populaire lyonnaise. Les enseignants, qui s'illustrent eux-mêmes dans le renouvellement doctrinal à l'œuvre à Lyon au tournant $\mathrm{du} \mathrm{XX}^{\mathrm{e}}$ siècle, oscillent entre objectifs pratique et éducatif du cours du droit. Les enseignements sont en rupture nette avec ce qui se fait encore dans les Facultés de droit. Ils se veulent plus proches des préoccupations quotidiennes des profanes du droit tout en restant fidèles aux innovations doctrinales de leur époque. L'importance accordée au droit social ou au droit constitutionnel est révélatrice de cette ambition tout à la fois pratique et moderne du cours de droit à destination de tous.

Mais, derrière les ambitions pédagogiques et doctrinales qui sont à l'œuvre à la Maison du peuple de la Croix-Rousse, apparaît également une conception politisée de l'enseignement du droit. Si les cours de droit professés par des grands noms de la doctrine juridique sont au service de l'auditoire profane, ils sont aussi, et surtout, au service plus discret d'une certaine conception de la République ${ }^{103}$.

$\mathrm{II}^{\circ}$ ) L'enseignement politique du droit, les cours de droit à l'Université populaire au service de la République démocratique, laïque et sociale

Le cours de droit à l'Université populaire procède-t-il d'une démarche purement désintéressée? De façon générale, l'enseignement populaire répond-il à une démarche seulement charitable ? Pour le cas de Lyon, la lecture des sources oblige à reconnaître qu'à l'Université populaire, même si le cours est construit sur la base d'une démarche sincère, il n'est pas moins dénué de visées politiques. Il est fondamental de révéler ces visées politiques pour comprendre le projet d'enseignement populaire du droit qui se forme au tournant du $\mathrm{XX}^{\mathrm{e}}$ siècle. On observe en effet qu'il y a une convergence radicale-socialiste des enseignements en droit (A) qui s'explique non pas au regard de l'auditoire profane mais au regard des stratégies personnelles des juristes qui enseignent à l'Université populaire (B). Ce décalage montre que derrière les vertus de l'enseignement populaire se dissimule une formation de réseaux au service du corps enseignant. L'Université populaire participe ainsi à la construction d'une certaine culture juridique dépendant de l'engagement politique de certains professeurs.

\footnotetext{
${ }^{98}$ Ibid., p. 98.

${ }^{99}$ Ibid., p. 49.

${ }^{100}$ Ibid., p. 4.

${ }^{101}$ AUPL, $\mathrm{n}^{\circ} 3$, p. 1.

${ }^{102} A U P L, \mathrm{n}^{\circ} 5$, p. 49. Il est probable que Lévy ait repris, dans une forme vulgarisée, les conclusions de sa thèse soutenue en 1896 sur le caractère essentiellement fiduciaire du droit de propriété (E. Lévy, La preuve par titre du droit de propriété immobilière, Paris, Pedone, 1896, 171 p.).

${ }^{103}$ F. Audren, «Les professeurs de droit, la République et le nouvel esprit juridique », in Mil neuf cent. Revue d'histoire intellectuelle, $\mathrm{n}^{\circ} 29$, 2011, p. 7-33 ; C. M. Herrera, «Anti-formalisme et politique dans la doctrine juridique sous la III ${ }^{\mathrm{e}}$ République », ibid., p. 145-165.
} 
A - La convergence radicale-socialiste des cours de droit : un programme politique pour un enseignement partisan

À l'Université populaire lyonnaise, nul ne doute que l'enseignement du droit procède de considérations politiques. Nul ne doute non plus que sa réception favorise un discours politique en faveur de l'État républicain en construction. Toutefois, il est frappant de constater que durant les quelques années de l'Université populaire, les enseignants ne revendiquent pas ouvertement les orientations politiques de leur enseignement, voire même, que l'Université populaire se targue d'une certaine neutralité politique ${ }^{104}$. Pourtant, une étude approfondie du contenu des enseignements démontre l'orientation politique de l'Université populaire. En effet, l'ensemble des cours, ceux de droit compris, convergent globalement vers la promotion du radicalisme - le secrétaire de l'Université populaire n'est autre qu'Herriot, figure centrale du radicalisme au XX $\mathrm{X}^{\mathrm{e}}$ siècle ${ }^{105}$. Entre 1899 et 1903, l'Université populaire lyonnaise couvre ainsi la période durant laquelle le Parti radical est au pouvoir, soit en participant au Gouvernement de défense républicaine de Waldeck-Rousseau, soit, après la victoire du bloc des gauches en 1902, avec le Gouvernement de Combes. Ce contexte n'est pas sans incidence, car on observe que l'Université populaire apporte son soutien aux politiques gouvernementales notamment en organisant, sous la coordination des enseignants en droit, des discussions sur les projets de $10 i^{106}$.

Cette convergence des enseignements vers un prisme radical-socialiste s'observe par les accents mis dans les cours de droit sur les thèses solidaristes. Il faut dire que le développement des Universités populaires coïncide avec l'épanouissement du solidarisme comme courant analytique rétrospectif de l'œuvre sociale de la Troisième République et comme programme prospectif du Parti radical ${ }^{107}$. Léon Bourgeois théorise le solidarisme à partir de 1896 avec la publication de La solidarité $e^{108}$ et en approfondit l'analyse jusqu'en $1902^{109}$, date à laquelle le sociologue radical Célestin Bouglé développe la théorie initiale en la perfectionnant ${ }^{110}$. C'est dans ce court espace chronologique que se situe une importante réception des thèses de Bourgeois, notamment parmi les juristes sensibles à la théorie du « quasi-contrat social »111. Cette réception s'observe dans les enseignements de droit à l'Université populaire. Dans l'introduction de sa conférence sur «La législation ouvrière et la démocratie », Paul Pic use du solidarisme :

\footnotetext{
${ }^{104}$ Bien que promoteur de cette «neutralité politique » de l'Université populaire, Édouard Herriot ne trompe personne : «L'Université populaire doit se défendre, au nom même des principes qui la fondent, d'intervenir dans le détail des affaires politiques, d'exercer, en temps d'élections, par exemple, une pression ou d'instituer une propagande. Elle travaille à mettre les hommes en état de juger, elle ne juge pas pour eux. En revanche, elle doit avoir ses idées politiques liées à son système philosophique ; il est bien évident qu'elle ne saurait éviter de se prononcer pour l'émancipation de l'individu, la moralisation de la société, la libération du travail, l'abolition des privilèges, l'extinction progressive des habitudes barbares comme celles de l'esclavage, de la réglementation de la prostitution et de la traite des blanches, du duel et de la guerre » (AUPL, $\mathrm{n}^{\circ}$ 5, p. 7).

105 S. Berstein, Édouard Herriot ou la République en personne, Paris, PFNSP, 1985, 327 p. et J. Henning, Le radicalisme d'Édouard Herriot, op. cit.

${ }^{106}$ AUPL, n ${ }^{\circ} 2$, p. 56.

${ }^{107}$ Sur l'importance du solidarisme dans la doctrine radicale : S. Audier, La pensée solidariste, Paris, PUF, 2010, p. 1-106 et S. Berstein, Histoire du Parti radical, t. 1, Paris, PFNSP, 1980, p. 37.

${ }^{108}$ L. Bourgeois, La solidarité, Paris, Armand Colin, 1896, 157 p.

${ }^{109}$ L. Bourgeois, «Les applications de la solidarité sociale », in RPP, $\mathrm{n}^{\circ}$ 91, 1902, p. 5-18.

${ }^{110}$ C. Bouglé, «L'évolution du solidarisme », in RPP, $\mathrm{n}^{\circ} 105,1903$, p. 480-505 et Le Solidarisme, Paris, V. Giard et E. Brière, 1907, 339 p.

111 M. Borgetto, «La doctrine solidariste de Léon Bourgeois : une nouvelle définition des rapports entre le politique, le social et le droit », in C. M. Herrera (dir.), Les juristes face au politique, t. 1, op. cit., p. 35-56 et C. Tixier, «La théorie du quasi-contrat social chez Léon Bourgeois. De l'État-association à la mutualisation », in Jus Politicum, n 15, 2015.
} 
« J'ai accepté avec empressement, car je suis un adepte convaincu de l'enseignement populaire, et je crois tout spécialement à l'utilité d'un enseignement populaire de la législation ouvrière, cette branche du droit née d'hier, et destinée à mettre plus de cœur, plus d'humanité et de solidarité véritable dans notre organisation sociale ! $»^{112}$.

Derrière l'usage des mots, les thèmes du solidarisme structurent les cours de Pic: analyse organiciste ${ }^{113}$, conclusion interventionniste ${ }^{114}$, réfutation de la thèse révolutionnaire pour la thèse évolutionniste ${ }^{115}$ et substitution du système assistanciel par le système assurantiel ${ }^{116}$. Devant un auditoire profane, Paul Pic assène une analyse solidariste du droit social dont les mots d'ordre sont issus la doctrine du Parti radical. Pic ne se cachait d'ailleurs pas de son adhésion au solidarisme. Les autres enseignements du droit usent du même prisme solidariste, que ce soit dans les enseignements de législation ouvrière ${ }^{117}$ ou de droit constitutionnel $^{118}$. Le bureau de l'Université populaire inscrit même l'institution toute entière dans la réalisation du programme solidariste en insistant sur son rôle dans la préparation des réformes législatives ${ }^{119}$.

Au-delà d'une coordination générale des enseignements selon la pensée solidariste, les cours de droit à l'Université populaire servent de manière plus concrète à soutenir les réformes préparées par les gouvernements Waldeck-Rousseau et Combes. On assiste ainsi, derrière les enseignements de droit public et de droit social, à une véritable propagande en faveur d'une promotion des lois sociales et laïques de la Troisième République. Paul Pic assume cette défense de l'œuvre de la Troisième République :

«Depuis le milieu de ce siècle, tous les vrais amis du peuple ont travaillé à édifier de toutes pièces une législation protectrice des travailleurs (...). Dès son avènement, et surtout depuis son triomphe définitif sur les partis réactionnaires, c'est-à-dire depuis 1880, la Troisième République a repris résolument le programme social de la République de $48 »^{120}$.

\footnotetext{
${ }^{112}$ AUPL, n ${ }^{\circ} 2$, p. 31.

113 «Dans une démocratie, en effet, le développement de la législation sociale est une nécessité organique, une condition de vie ou de mort pour le régime ; une démocratie n'est digne de ce nom et ne mérite de vivre qu'à la condition de travailler sans cesse à l'amélioration du sort de tous, sans préoccupation de classes ni de castes » (ibid., p. 36).

114 «C'est à l'économie sociale aussi généreuse et démocratique dans son essence que l'économie politique classique était égoïste et aristocratique, qu'il appartient de guider le législateur, de lui frayer la route, en lui signalant les écueils et en lui montrant le but ; but noble et haut entre tous, puisqu'il ne s'agit de rien de moins que d'assurer la paix sociale, par la coopération harmonieuse de toutes les forces productrices de la nation, sous l'égide de l'État! » (ibid., p. 35).

115 «Décréter d'autorité la suppression du salariat et la généralisation du régime coopératif. Ce serait là une véritable révolution sociale ; or, je ne crois pas beaucoup, je l'avoue, à l'efficacité des révolutions sociales, surtout des révolutions limitées à un pays isolé (...). Je crois, au contraire, fermement à l'évolution de l'industrie dans le sens d'une participation de plus en plus large des travailleurs au produit net de l'entreprise. Je crois au développement des services municipaux, et à la mainmise par les villes sur les entreprises d'intérêt collectif (services d'eau, de gaz, peut-être même de transport comme dans maintes villes d'Angleterre ou d'Amérique). Mais toute évolution est lente et elle doit être lente pour être définitive et féconde » (ibid., p. 42).

116 «L'on ne saurait nier la supériorité de l'assurance sociale, qui respecte la dignité du travailleur, sur l'assistance, de la solidarité sur la charité, cet idéal de l'Église : non pas de l'Église des premiers âges, hardiment égalitaire et niveleuse, mais de l'Église des temps modernes, qui cherche son point d'appui dans l'aristocratie de fortune, comme elle le cherchait naguère dans l'aristocratie de naissance, et qui par conséquent a intérêt à maintenir les classes pauvres dans une situation de dépendance, dont seul l'État laïque peut les affranchir par l'assurance, et par toute une législation sociale, à la foi (sic) protectrice et égalitaire ! » (ibid., $\mathrm{n}^{\circ} 2$, p. 47).

${ }^{117}$ AUPL, $\mathrm{n}^{\circ}$ 3, p. 106.

${ }_{118} A U P L, \mathrm{n}^{\circ}$ 4, p. 5.

119 Édouard Herriot : «nous sommes à un tournant de l'histoire ; sous l'impulsion d'un petit nombre d'hommes les plus éclairés et les plus généreux, les idées de solidarité sont en train de passer de la théorie dans la pratique, des manuels de philosophie dans la vie. Aucune des soi-disant classes de la société ne peut désormais rester isolée (...). C'est tous ensemble que les hommes doivent étudier les réformes nécessaires pour fonder la société de l'avenir, c'est tous ensemble qu'ils ont à rechercher ce qui peut faire régner entre eux la justice et la paix sociale vers lesquelles tendent tous nos efforts $\gg\left(A U P L, \mathrm{n}^{\circ} 5, \mathrm{p} .14\right)$.
}

${ }^{120} A U P L, \mathrm{n}^{\circ} 2$, p. 35 . 
Pour le professeur de droit, il s'agit ainsi de préparer les réformes en cours au sein du gouvernement Waldeck-Rousseau. Après avoir plaidé en faveur de la création d'une caisse de secours social et du développement des bureaux de placement pour lutter contre le chômage, il conclut :

« Le programme social que je viens d'esquisser est celui-là même dont le ministère actuel travaille, avec le concours du Parlement, à assurer la réalisation : vous savez en effet que M. Waldeck-Rousseau a inscrit, au premier rang des projets à maintenir à l'ordre du jour, le projet sur les Caisses de retraites ouvrières $»^{121}$.

La teneur politique du cours de droit ne saurait être plus explicite - Paul Pic ajoute tout de même une revendication personnelle: la nécessité d'élaborer un «code ouvrier ${ }^{122}$. D'ailleurs, la défense du projet de Waldeck-Rousseau en faveur des retraites ouvrières, à défaut d'aboutir à la Chambre, est en discussion permanente à l'Université populaire tout au long de l'année 1901, avec des débats organisés par Chazette pour expliquer l'intérêt du projet gouvernemental $^{123}$. De la même manière, l'Université populaire organise des cours explicatifs sur le projet de réforme des syndicats professionnels déposé par le ministre Alexandre Millerand au Parlement le 14 novembre 1899 qui donnera la loi sur les associations du $1^{\mathrm{er}}$ juillet $1901^{124}$. Ainsi, derrière un discours professoral, les enseignements en droit à l'Université populaire fluctuent entre défense de l'œuvre de la Troisième République et préparation aux réformes à venir. Après la victoire du bloc des gauches en 1902, ce phénomène de politisation des cours s'observe à propos de la loi de séparation des Églises et de l'État. Durant deux années, les enseignants, fidèles à la doctrine radicale du Gouvernement, tentent de convaincre l'opinion lyonnaise des bénéfices du régime de laïcité. À Paul Pic qui plaide pour «l'État laïque ${ }^{125}$, s'ajoutent Jean Appleton qui consacre en 1902 son cours de droit public à «la liberté de conscience ", notion au cœur de l'article $1^{\text {er }}$ du projet de Séparation ${ }^{126}$, et Marius Moutet qui accorde une conférence sur «les idées religieuses et l'assistance sociale ${ }^{127}$. Cette préparation à la séparation entre les Églises et l'État donne lieu à tout un programme structuré durant l'année 1903 autour de l'organisation du centenaire d'Edgar Quinet présenté comme précurseur du régime laïc ${ }^{128}$. Ainsi, sans surprise, l'auditoire de l'Université populaire assiste à la défense des différentes réformes législatives entreprises par les radicaux au Gouvernement et au Parlement. Cette défense du programme radical en général s'observe encore dans la promotion de l'impôt sur le revenu ou de la jurisprudence du juge Magnaud par Jean Appleton ${ }^{129}$.

Cet aspect radical-socialiste des enseignements à l'Université populaire se constate encore dans le refus des thèses socialistes. Même si Emmanuel Lévy et Marius Moutet enseignent à l'Université populaire, ceux-ci font office d'exception car la plupart des autres cours de droit dénoncent les thèses socialistes. Ce refus du socialisme passe notamment par les discussions envisagées comme scientifiques dans plusieurs enseignements entre «thèse de la révolution » et «thèse de l'évolution ». Les thèses sur «l'évolutionnisme institutionnel », appuyées sur une analyse sociale des théories darwiniennes, se développent dans les milieux radicalisant à la fin du XIX ${ }^{\mathrm{e}}$ siècle $^{130}$. Cette approche se traduit dans nombre d'enseignements :

\footnotetext{
${ }^{121}$ Ibid., p. 47.

122 Ibid., p. 49.

${ }^{123}$ Ibid., p. 56.

${ }^{124}$ Ibid.

${ }^{125}$ Ibid., p. 47.

${ }^{126}$ AUPL, $\mathrm{n}^{\circ} 4$, p. 3.

${ }^{127}$ Ibid., $\mathrm{n}^{\circ} 5$, p. 100.

${ }^{128}$ Ibid., p. 57.

${ }^{129} \mathrm{Ibid} . \mathrm{n}^{\circ}$ 2, p. 12 et 66. Marie-Anne Frison-Roche a montré le soubassement radical-socialiste dans l'action du juge Magnaud (M.-A. Frison-Roche, «Le modèle du bon juge Magnaud », op. cit., p. 338).

${ }^{130} \mathrm{~J}$. Henning, Le radicalisme d'Édouard Herriot, op. cit., p. 80-82.
} 
Pic $^{131}$, Chazette ${ }^{132}$ ou encore Appleton ${ }^{133}$ plaident pour les bienfaits de l'évolution en dénonçant les méfaits de la révolution. Cet aspect est encore renforcé par l'accueil de Ferdinand Buisson, partisan de la thèse de l'évolution comme élément constitutif du radicalisme ${ }^{134}$, lors d'une soirée de l'Université populaire ${ }^{135}$.

Cette convergence radicale des enseignements juridiques à l'Université populaire lyonnaise marque en partie une rupture avec l'enseignement dispensé en même temps à la Faculté de droit. Il semble que les professeurs qui donnent quelques heures de leur temps à la Maison du peuple trouvent une liberté de parole moins affirmée au Palais de l'Université où l'institution modère inconsciemment les propos politiques. Ce caractère partisan des enseignements est consubstantiel au projet des Universités populaires et au contexte dans lequel elles s'inscrivent. Au niveau local, il faut remarquer que ce caractère politique sert autant le projet pédagogique de l'Université populaire que les stratégies personnelles des enseignants.

B - L'engagement politique des professeurs à l'Université populaire : l'enseignement du droit permettant l'implication politique du juriste en société

Entre les lignes des programmes et des comptes-rendus de cours conservés aux Annales de l'Université populaire lyonnaise, une question demeure : quelles sont les motivations des enseignants qui viennent, de façon bénévole, donner plusieurs soirées de cours à l'Université populaire ? Une première réponse, dont on ne saurait douter, est que les professeurs sont convaincus de la nécessité de dispenser des cours de droit à un public profane. Mues par des préoccupations philanthropiques, les convictions de ces juristes sont indissociables de l'engagement dreyfusard de rapprochement des élites et du peuple. Mais on ne saurait se contenter de cette lecture après l'observation des parcours extra-universitaires de ces enseignants. En effet, on constate qu'avec l'Université populaire se dessinent des stratégies partisanes pour chacun de ces enseignants. Sans remettre en cause la sincérité de l'engagement associatif des juristes à l'Université populaire, il faut remarquer que celui-ci sert leur carrière. À bien des égards, l'Université populaire constitue pour les juristes un lieu de sociabilité politique et de mise en réseau utiles au-delà des seules années dreyfusiennes.

Pour s'en convaincre, il suffit d'observer l'engagement politique des différents enseignants qui participent aux activités de la Maison du peuple. Presque tous militent à la même époque au Parti radical. D'ailleurs, leur expertise juridique semble appréciée par le Comité radical du Rhône qui les mobilise pour diverses élections. Jean Appleton, vice-président de l'Université populaire, est élu en 1900 conseiller municipal sur la liste du futur maire Victor Augagneur ${ }^{136}$ - ce dernier étant devenu entre-temps Président d'honneur de l'Université populaire. Pour la campagne municipale de 1904, on retrouve encore plusieurs enseignants inscrits sur la liste d'union entre radicaux et républicains-socialistes. Parmi les élus, Justin Godart et Édouard Herriot font leur entrée au Conseil municipal ${ }^{137}$ - début d'une longue carrière politique pour les deux amis. Ils sont rejoints en 1905 par Paul Pic élu conseiller municipal lors d'une élection partielle ${ }^{138}$ après avoir échoué au Conseil général en $1901^{139}$. En 1908, c'est au

\footnotetext{
${ }^{131} A U P L, \mathrm{n}^{\circ} 2$, p. 35-36, 42.

132 Ibid., p. 56.

${ }^{133}$ Ibid., p. 12.

${ }^{134}$ F. Buisson, La politique radicale, Paris, Giard et Brière, 1908, p. 1-4.

${ }^{135}$ AUPL, $\mathrm{n}^{\circ} 3$, p. 27.

${ }^{136}$ AML, 1217WP160, p. 3.

${ }^{137}$ AML, 1217WP166, p. 3.

${ }^{138}$ AML, 1217WP167, p. 130.

${ }^{139}$ D. Bayon et L. Frobert, «Lois ouvrières et réformisme social dans l'œuvre de Paul Pic (1862-1944)», op. cit., p. 53.
} 
tour de Louis Chazette de rejoindre le Conseil municipal sur la liste radicale conduite par le maire sortant Édouard Herriot ${ }^{140}$. Chazette sera élu $3^{\mathrm{e}}$ adjoint au maire en $1912^{141}$ et $2^{\mathrm{e}}$ en $1919^{142}$. Du côté des enseignants socialistes, on observe le même parcours sous des listes dirigées par Herriot : Lévy est élu conseiller municipal en $1912^{143}-$ il devient même $1^{\text {er }}$ adjoint en $1919^{144}$ - et Moutet intègre l'équipe du maire en $1919^{145}$. À l'opposé de ces engagements sous les listes municipales d'union de la gauche, il faut relever le cas particulier de Paul Huvelin qui s'engage en devenant tête de liste de l'union de la droite dans le $7^{\mathrm{e}}$ arrondissement pour l'élection municipale de 1919 puis Président de la Fédération républicaine du Rhône ${ }^{146}$. L'importance des engagements politiques des enseignants à l'Université populaire dans les années qui suivent leurs participations témoigne de l'existence d'un lieu de sociabilité politique. Paul Pic, Justin Godart, Louis Chazette, Jean Appleton et Emmanuel Lévy intègrent l'entourage politique de Victor Augagneur et d'Édouard Herriot. Les proximités sont encore plus frappantes lorsque l'on s'intéresse aux élections législatives. Justin Godart est élu député en 1906 et devient ministre en 1915. Marius Moutet devient député en 1914 et Louis Chazette est élu sur la liste radicale aux législatives de 1924 - contre Huvelin qui se présentait sur une liste poincariste. Jean Appleton se présente lui aussi aux législatives sur la liste radicale conduite par Herriot en 1924 mais sans être élu. Il faut ajouter que deux autres enseignants de l'Université populaire, non juristes, sont élus sénateurs : Édouard Herriot bien sûr, élu sénateur en 1912, mais aussi Georges Beauvisage, élu sénateur radical en 1909.

Ainsi, il est frappant de constater que parmi la vingtaine d'enseignants permanents à l'Université populaire, près d'un quart accède à une fonction élective nationale et que trois d'entre eux deviennent ministres. L'Université populaire constitue alors, entre enseignants, un de ces lieux de sociabilité politique où se reconstitue la vie publique lyonnaise après les troubles de l'affaire Dreyfus. Les juristes lyonnais participent pleinement à cette sociabilité politique dont il ne faut pas minimiser les effets sur leur propre production doctrinale. Cette mise en réseau autour du personnel politique permet aux professeurs de droit de sortir de l'Université en intégrant des postes décisionnels de premier plan ou en obtenant des consultations juridiques commandées par la mairie. Ici, la mise en réseau produit ses effets : les professeurs de droit sont nommés par Herriot à certaines fonctions administratives complémentaires de leur activité à la Faculté en raison de leur expertise juridique. Pour Pic, Lévy et Godart, la municipalité s'appuie sur leurs travaux relatifs à la régie directe pour assurer une politique de socialisme municipal ${ }^{147}$. Pour le développement du logement social dans l'agglomération lyonnaise, là encore les juristes intègrent les différents établissements d'HBM à des fonctions de direction en raison de leur expertise juridique (pour Pic, Lévy et Josserand) ${ }^{148}$. Ainsi, l'Université populaire sert à l'élaboration de réseaux de sociabilité politique mélangeant expertise universitaire et engagement partisan.

Cette mise en relation d'une génération de juristes avec le Parti radical s'inscrit dans la dynamique générale de la constitution des groupes d'intérêts à la sortie de la crise dreyfusienne. Les stratégies partisanes des juristes enseignant à l'Université populaire ne peuvent en effet se comprendre que dans l'environnement général dans lequel elles s'inscrivent. L'Université populaire lyonnaise catalyse alors des engagements associatifs qui dépassent la seule sphère

\footnotetext{
${ }^{140}$ AML, 1217WP170, p. 3.

${ }^{141}$ AML, 1217WP174, p. 5.

142 AML, 1217WP179, p. 7.

143 AML, 1217WP174, p. 3.

144 AML, 1217WP179, p. 7.

145 Ibid., p. 5.

${ }^{146}$ F. Audren, « Paul Huvelin (1873-1924) : juriste et durkheimien », op. cit., p. 123-124.

${ }^{147}$ J. Henning, Le radicalisme d'Édouard Herriot, op. cit., p. 136-140.

148 J. Henning, «Le logement social à Lyon de 1906 à 1928. Principes et mise en œuvre d'un service public municipal des habitations à bon marché », in Droit et ville, ${ }^{\circ}$ 89, 2020, p. 111-127.
} 
académique. En cela, les cours de droit à l'Université populaire obéissent à une synergie qui mêle engagement politique, expertise juridique, positionnement académique et engagement associatif. Notamment, les enseignants de la Maison du peuple ne sont jamais très éloignés de la Ligue des droits de l'Homme. Conséquence directe de l'affaire Dreyfus, la Ligue catalyse à Lyon dès 1898 l'engagement dreyfusard. Les membres fondateurs de la section lyonnaise de la Ligue des droits de l'homme et ceux de l'Université populaire sont sensiblement les mêmes : Lépine, Augagneur, Herriot, Appleton, Chazette, Desparmet-Ruello, Godart, Moutet ${ }^{149}$. Chazette, Moutet et Appleton appartiennent au service contentieux de la Ligue et, en qualité d'avocats, représentent l'association en justice. Appleton devient même, après Lépine, Président de la section de Lyon de 1906 à $1909^{150}$. La Ligue des droits de l'Homme porte un projet politico-juridique d'un genre nouveau alliant soutien à des cas individuels d'injustice et intervention politique ${ }^{151}$. Or, ces deux modes d'intervention se fondent sur le mythe de continuité historique entre la Révolution et la Troisième République. Les principes énoncés dans la Déclaration des droits de l'homme et du citoyen de 1789 doivent être diffusés et défendus par l'association tant face aux juges que face au législateur ${ }^{152}$. Cette intégration nouvelle de la Déclaration de 1789 dans la conception républicaine de la justice et du droit n'est pas sans incidence sur le contenu des enseignements à l'Université populaire. Jean Appleton, qui est par ailleurs à l'origine de l'affichage dans toutes les écoles de Lyon de la Déclaration des droits de l'Homme ${ }^{153}$, utilise les cours qu'il professe à l'Université populaire pour promouvoir un usage du texte de 1789 en République ${ }^{154}$. À certains égards, cet engagement des enseignants de droit au sein de la Ligue des droits de l'homme participe au passage de l'État légal à l'État de droit qui s'opère alors dans l'espace doctrinal ${ }^{155}$.

Poursuivant la défense d'une conception républicaine du droit et de la justice, l'Université populaire lyonnaise mobilise encore ses membres juristes à l'occasion de l'accueil de la Fédération abolitionniste internationale. Cette organisation, fondée en 1875, regroupe les militants anti-prostitution ${ }^{156}$. Mais en plus de la lutte pour l'abolition de la prostitution, la Fédération abolitionniste s'illustre au tournant $\mathrm{du} \mathrm{XX}^{\mathrm{e}}$ siècle par des campagnes contre les pratiques abusives de la police des mœurs ${ }^{157}$. Soutenue par Victor Augagneur, la Fédération regroupe dans le Rhône des juristes engagés - encore Jean Appleton et Marius Moutet - qui reçoivent à l'Université populaire pour quelques conférences les spécialistes du régime juridique de la prostitution ${ }^{158}$. Parmi les conférenciers qui viennent discuter du droit de la prostitution, on trouve surtout Paul Appleton - frère cadet de Jean et futur professeur de droit à la Faculté de Lille - qui soutient en 1903 sa thèse de droit sur La traite des blanches ${ }^{159}$. Là

\footnotetext{
${ }^{149}$ E. Naquet, La Ligue des droits de l'Homme : une association en politique (1898-1940), thèse soutenue à l'IEP de Paris, 2005, p. 131-132.

${ }^{150}$ C. Fillon, Histoire du Barreau de Lyon, op. cit., p. 72-88.

${ }^{151}$ D. Lochak, «Le droit au service des droits de l'homme », in Les évènements fondateurs, l'affaire Dreyfus, op. cit., p. 186-188.

${ }^{152}$ E. Naquet, «L'universalisme des droits de l'homme et du citoyen », in ibid., p. 102-110.

${ }^{153}$ AML, 1217WP160, p. 104.

${ }^{154} A U P L, \mathrm{n}^{\circ} 5$, p. 4.

155 M.-J. Redor, De l'État légal à l'État de droit. L'évolution des conceptions de la doctrine publiciste française (1870-1914), Aix-en-Provence, PUAM, 1992, 330 p.

${ }^{156} \mathrm{H}$. Duffuler-Vialle, L'évolution de la réglementation de la prostitution durant l'entre-deux-guerres : l'exemple du Nord de la France, Paris, Mare et Martin, 2017, p. 25-60.

157 P. Vielfaure, «La Cour de cassation et le réglementarisme (1800-1946) : la jurisprudence de la Chambre criminelle relative à l'inscription et aux visites sanitaires imposées aux prostituées », in A. Leca et F. Vialla (dir.), Le risque épidémique. Droit, histoire, médecine et pharmacie, Aix-en-Provence, PUAM, 2003, p. $247-263$.

${ }^{158}$ AUPL, $\mathrm{n}^{\circ} 5$, p. 112.

${ }^{159}$ P. Appleton, La traite des blanches, Paris, Rousseau, 1903, 299 p. La thèse de Paul Appleton s'inscrit dans le contexte particulier consécutif de l'affaire de la Traite des blanches de Bruxelles (J.-M. Chaumont et C. Machiels (dir.), Du sordide au mythe. L'affaire de la traite des blanches (Bruxelles, 1880), Louvain-la-Neuve, Presses universitaires de Louvain, 2013, 268 p.).
} 
encore, les conférences de droit sont l'occasion de diffuser, au sein de la population lyonnaise, un programme sanitaire et moral en faveur de la disparition de la prostitution ${ }^{160}$ et de construire un réseau de juristes, de médecins et d'hommes politiques autour de revendications communes.

Dans des proportions moindres, on constate aussi que l'Université populaire lyonnaise catalyse d'autres groupes de réflexion. Louis Josserand, lors des réunions de fondation de l'Université populaire, n'intervient pas en tant que professeur de droit, mais comme président $\mathrm{du}$ «cercle positiviste Le Chêne ${ }^{161}$. Jean Appleton, qui cumule les engagements politicoassociatifs, se présente lui aussi comme vice-président du Caveau lyonnais, société de chansonniers masculins aux ramifications politiques qui organise des concerts à la Maison du peuple ${ }^{162}$. Dans ces diverses associations, on note aussi la présence d'Adrien Storck, lui aussi membre de l'Université populaire ${ }^{163}$, qui possède la maison d'édition chargée de la publication des Annales et, dans le paysage éditorial lyonnais, de la plupart des publications proches du radicalisme - il est coéditeur de la revue Questions pratiques de législation ouvrière et de différentes publications de la Faculté de droit - et des professeurs lyonnais participant à l'Université populaire - Lacassagne, Lévy, Lépine, Bertrand, Charléty. Enfin, il faut noter, l'articulation de l'Université populaire lyonnaise avec son environnement géographique plus large. Les cours de droit professés à la Croix-Rousse ont vocation à s'exporter dans la construction de réseaux plus amples. Dans les différents quartiers de la ville, Appleton à Vaise $^{164}$ et Chazette à Perrache ${ }^{165}$, l'Université réplique ses enseignements dans les cercles populaires, associations affiliées au socialisme, et dans les « groupes d'études sociales » affiliés aux leplaysiens. Mais au-delà de Lyon, l'Université populaire crée une filiale à Rive-de-Gier ${ }^{166}$ et prononce des « conférences suburbaines du dimanche » dans toute la région - y participent : Emmanuel Lévy, Marius Moutet, Justin Godart, Édouard Herriot et Louis Chazette ${ }^{167}$. Appleton quant à lui se montre téméraire en répliquant son cours de libertés publiques à Saint-Claude dans le Jura ${ }^{168}$. Ce réflexe d'expansion et de diffusion se comprend dans l'environnement général des Universités populaires au tournant du $\mathrm{XX}^{\mathrm{e}}$ siècle. Une lutte discrète a lieu entre les universités populaires à tendance socialiste et celles à tendance radicale. À Lyon, les principaux acteurs du projet d'enseignement populaire tentent de rattacher leur Université populaire à celle du Faubourg Saint-Antoine de Paris ${ }^{169}$. Mettre en réseau les Universités populaires en exportant les cours constitue alors un enjeu de propagande politique. L'Université populaire lyonnaise reçoit ainsi des conférenciers parisiens, comme Ferdinand Buisson et Henry Michel ${ }^{170}$.

Sans que l'on sache vraiment pourquoi, l'aventure de l'Université populaire lyonnaise s'arrête brutalement à la rentrée de septembre 1903. Pourtant, les cours de droit à destination de tous ne s'arrêtent pas à Lyon. La plupart des membres actifs de l'Université populaire - Pic, Godart, Appleton, Herriot, Lévy - se rejoignent dans la création de l'Office social de renseignements et d'études de Lyon. Cet Office, initié par Pic et Godart dans la suite de l'Université populaire et de la fondation des Questions pratiques de législation ouvrière, devient un lieu d'enseignement populaire des sciences sociales en général. Les origines intellectuelles de cet Office actif entre 1901 et 1906 ont été discutées pour conclure à une

\footnotetext{
${ }^{160} A U P L, \mathrm{n}^{\circ} 5$, p. 7.

${ }^{161} A U P L, \mathrm{n}^{\circ} 1$, p. 10.

${ }^{162} A U P L, \mathrm{n}^{\circ} 4$, p. 75.

${ }^{163} A U P L, \mathrm{n}^{\circ} 1, \mathrm{p} .10$.

${ }^{164} A U P L, \mathrm{n}^{\circ} 2$, p. 12.

${ }^{165}$ AUPL, n ${ }^{\circ} 4$, p. 38-43.

${ }^{166} A U P L, \mathrm{n}^{\circ} 5$, p. 103.

${ }^{167}$ Ibid., p. 110.

${ }^{168}$ AUPL, $\mathrm{n}^{\circ}$ 4, p. 53.

${ }^{169} A U P L, \mathrm{n}^{\circ} 1, \mathrm{p} .5$.

${ }^{170} A U P L, \mathrm{n}^{\circ} 3$, p. 13 et $\mathrm{n}^{\circ} 5$, p. 52-77.
} 
filiation leplaysienne ${ }^{171}$. Si la méthode prônée par Le Play de l'étude monographique est au cœur du projet de l'Office social ${ }^{172}$, l'institution procède d'une philosophie différente ${ }^{173}$ - à cet égard il est exagéré d'affirmer la filiation leplaysienne ${ }^{174}$. L'Office social semble davantage s'inscrire dans la continuité de l'Université populaire lyonnaise. En 1906, il se transforme en «Collège libre lyonnais des sciences sociales », une institution orientée vers l'enseignement grâce au soutien financier accordé par la municipalité dirigée par Herriot ${ }^{175}$. Inspiré par le Collège libre des sciences sociales de Paris, l'établissement lyonnais regroupe encore les juristes qui avaient participé à l'Université populaire ${ }^{176}$. La tradition des cours de droit à destination de tous y est maintenue selon les mêmes principes qu'à l'Université populaire : primauté du droit social et du droit constitutionnel, exclusion des autres branches du droit ${ }^{177}$. Dans une perspective peu éloignée, les cours de droit à destination de tous sont encore prolongés après l'expérience de l'Université populaire par l'intervention de la mairie. Reprenant une institution ancienne ${ }^{178}$, Herriot décide de créer des cours municipaux de droit en $1907^{179}$. Ces cours du soir obéissent encore au projet initial de l'Université populaire en sélectionnant un

171 B. Sylvestre, Éducation populaire et mouvements de jeunesse laïques à Lyon sous les mandats d'Édouard Herriot (1896-1957), thèse soutenue à l'Université Lyon II, 2017, p. 313-319.

${ }^{172}$ N. Hakim, «Une revue lyonnaise au cour de la réflexion sur le droit social : les Questions pratiques de législation ouvrière et d'économie sociale », op. cit., p. 134.

${ }^{173}$ L. Guerlain, L'école de Le Play et le droit : contribution à l'histoire des rapports entre droit et science sociale, Issy-les-Moulineaux, LGDJ, 2017, p. 236.

${ }^{174}$ Les deux animateurs principaux de l'Office, Pic et Godart, n'ont jamais montré leur adhésion aux thèses leplaysiennes. Dans son Traité élémentaire de législation industrielle, Pic expliquait son opposition aux doctrines leplaysiennes : «Il est facile de comprendre comment cette école unique, l'école historique, procédant d'une méthode rigoureusement expérimentale, ont pu sortir trois écoles nettement caractérisées : l'école du socialisme d'État avec ses nombreuses variétés et ses rameaux dissidents, tel que le socialisme chrétien ; l'école chrétienne de Le Play, aux tendances libérales, et l'école positiviste contemporaine, éclectique ou sociologique, à laquelle nous nous rallions (...). L'école de Le Play nous paraît d'un optimisme excessif, (...) quand elle prétend que le régime corporatif sous le patronage chrétien, et subsidiairement la coopération, suffiront à rétablir l'harmonie entre le capital et le travail, et que l'État n'a que faire dès lors, d'intervenir pour protéger l'ouvrier, tout au moins l'ouvrier adulte. L'expérience démontre en effet que l'initiative privée est impuissante à réaliser des réformes aussi complexes, que celles dont les abus de l'industrialisme ont révélé l'urgence. Pour un patron qui se conformera au devoir moral de solidarité, dix peut-être ne s'y conformeront pas. Et nul ne saurait leur en faire un crime, attendu qu'ils sont eux-mêmes, bien souvent, les victimes de la loi implacable de la concurrence. » (P. Pic, Traité élémentaire de législation industrielle, op. cit., 1894, p. 21-25). Les thèses de la coopération chères à Justin Godart semblent quant à elles se rattacher au fouriérisme, très influent dans les milieux lyonnais.

175 Questions pratiques de législation ouvrière, 1906, p. 65.

${ }^{176}$ F. Audren, «Le droit au service de l'action. Éléments pour une biographie intellectuelle d'Emmanuel Lévy (1871-1944) », in Droit et sociétés, ${ }^{\circ}$ 57, 2004, p. 99.

${ }^{177}$ Lors de l'inauguration, Paul Pic résume les enseignements qui se déclinent sur plusieurs années : « l'ensemble des questions ouvrières (juridictions industrielles; travail des enfants, des femmes et des adultes, travail à domicile ; apprentissage et enseignement technique, contrat de travail ; mutualité, coopération, logements ouvriers, assistance sociale ; monopoles, trusts, régies d'État ou régies municipales). Puis l'on abordera la législation fiscale, le droit constitutionnel et électoral » (Questions pratiques de législation ouvrière, 1906, p. 66). Pour la première année, les cours sont assurés par Charles Brouilhet (droit social et droit fiscal) et par Paul Pic (droit social) (Ibid., p. 65). Pour la seconde année les cours sont : «Accidents du travail » (Bigallet, docteur en droit) ; «Les syndicats professionnels » (Galland, chargé de conférences à la Faculté) ; «Les assurances sociales » (Amieux, chargé de conférences à la Faculté) ; «Les doctrines sociales » (Lévy, professeur à la Faculté) (Ibid., p. 67).

${ }^{178}$ Les cours municipaux du soir sont développés à Lyon durant la décennie 1870. Pour le droit, on note la création, en 1872 d'un cours «de morale sociale» pour lequel «le professeur enseignera, ce que malheureusement on n'ignore trop en France, les droits et les devoirs du citoyen, les lois morales, qui s'imposent à toute la société ; il appréciera les constitutions qui régissent les différents pays » (AML, 2CM7, p. 336 et Le journal de Lyon, 11 avril 1872). Ce cours suscita la désapprobation de l'Assemblée nationale qui interpella le Gouvernement pour demander la suspension du cours en raison de sa tonalité trop républicaine, «irréligieuse et athée » (L'univers, 8 mai 1872). En 1873, un cours d'économie politique est créé puis attribué, en 1881, à Adrien Audibert, professeur à la Faculté de droit.

${ }^{179}$ AML, 1217WP169, p. 61. 
programme pratique mais au contenu politique. Un cours d'" histoire des doctrines économiques et sociales » confié à Charles Brouilhet dissimule en réalité un enseignement de législation ouvrière. Cette fois la municipalité fixe le programme du cours : rémunération du capital et du travail, salariat, association, protection du travailleur, assurance et assistance sociales, question de l'impôt global et progressif sur le revenu, monopoles, monopole d'État, régie municipale et concessions ${ }^{180}$. Ce cours dispensé à la Faculté de droit est complété par un cours de «Finances publiques municipales » créé en 1910 et confié à Émile Bouvier, juriste proche de la municipalité ${ }^{181}$.

Ainsi, l'enseignement du droit à l'Université populaire lyonnaise s'inscrit dans un environnement général complexe qui mêle lieu de sociabilité politique et stratégies personnelles des enseignants. Cet environnement mis en lumière par l'engagement des professeurs de droit à la Maison de peuple doit être pris en compte pour comprendre d'une part le renouvellement de la doctrine qui s'opère à la charnière des $\mathrm{XIX}^{\mathrm{e}}$ et $\mathrm{XX}^{\mathrm{e}}$ siècles ${ }^{182}$ et d'autre part la conception naissante d'un enseignement du droit à destination de tous. À bien des égards, le projet pédagogique de l'Université populaire sert autant l'auditoire profane que l'enseignant spécialisé. On peut tout de même s'interroger sur la spécificité de l'Université populaire par rapport aux autres groupes où se côtoient les mêmes acteurs engagés. Il est difficile de dire en quoi l'Université populaire se distinguerait du Parti radical, de la Ligue des droits de l'Homme, de la Fédération abolitionniste ou d'autres groupes d'intérêt. L'ensemble de ces structures participent à la formation d'un microcosme politique aux multiples facettes auquel participent de nombreuses personnalités liées par des réseaux locaux. Néanmoins, parmi cet ensemble complexe, l'Université populaire préfigure l'une des évolutions les plus notables de la vie politique de l'entre-deux-guerres : le remplacement dans la classe politique du personnel issu des professions libérales par un personnel issu de la fonction publique et en particulier du corps enseignant. Le cas de Lyon montre que cette « république des professeurs ${ }^{183}$, qui accède aux fonctions en 1924 avec Herriot, est déjà en germe dans le réseau des Universités populaires.

* $* \quad *$

L'enseignement du droit à l'Université populaire répond ainsi à deux caractéristiques : un enseignement en rupture avec les matières traditionnellement enseignées à la Faculté de droit et un enseignement au contenu politisé. Cette double caractérisation se comprend par rapport aux stratégies personnelles du corps enseignant à l'égard de leur positionnement doctrinal et à l'égard de la formation de réseaux de sociabilité politique et associative. Mais, au-delà de ces observations demeure une question : quel est l'effet réel des enseignements en droit sur l'auditoire profane, sensément ouvrier, auquel ils s'adressent ? Les rapports annuels publiés par l'Université populaire sont ambigus. Ils montrent que, pour la première année, les cours de législation ouvrière de Louis Chazette sont suivis chaque soir par 50 auditeurs réguliers ${ }^{184}$. Le nombre est important en lui-même mais faible comparé à la moyenne de 132 auditeurs pour les autres cours ${ }^{185}$ et dérisoire comparé au cours le plus suivi, celui de littérature d'Herriot, qui accueille chaque jeudi 220 auditeurs. Ainsi, les cours de droit sont parmi ceux qui sont les moins suivis ; seuls les cours de mathématiques font pire que le droit ${ }^{186}$. On ignore les causes de cette

\footnotetext{
180 Ibid.

${ }^{181} \mathrm{~J}$. Henning, Le radicalisme d'Édouard Herriot, op. cit., p. 140.

${ }^{182}$ N. Hakim et F. Melleray, Le renouveau de la doctrine française, op. cit., p. 2-6.

183 A. Thibaudet, La République des professeurs, Paris, Grasset, 1927, 264 p.

${ }^{184}$ AUPL, $\mathrm{n}^{\circ} 2$, p. 86.

185 Ibid., p. 55.

${ }^{186}$ Ibid., p. 86.
} 
désaffection - la personnalité de l'enseignant, la prégnance d'une approche trop doctrinale du droit, le caractère peu divertissant du cours - d'autant que dans le quartier Perrache, les cours de Chazette sont quant à eux suivis par près de 200 auditeurs ${ }^{187}$. À vrai dire, on ne dispose pas de sources permettant d'évaluer l'intérêt que le public tire des cours populaires de droit. Seul un témoignage est resté, celui de Claudine Simple qui suivait de manière assidue les cours de droit de Chazette qu'elle évoquait en ces termes :

«Il voit très clair, je vous l'affirme, dans ces questions qui nous touchent tout particulièrement. Il ne prend pas l'intérêt du patron contre les ouvriers, bien qu'il appartienne certainement à une famille bourgeoise, mais il est pour la justice, pour le droit de chacun. Il nous a montré que tout l'avenir de la question ouvrière est dans l'organisation des syndicats professionnels et qu'il faudra bien que les ouvrières se syndiquent, car c'est toujours sur elles que l'on compte, sur leur faiblesse pour maintenir le bas prix des salaires; on les exploite encore plus que les hommes. Il nous a beaucoup intéressés sur la question des coopératives de consommation, car il y en a depuis longtemps à la Croix-Rousse, coopératives pour le vin, le pain, le charbon, l'épicerie ; nous y avons pris des parts et nous nous en trouvons très bien (...). Il nous a parlé de toutes les réformes qui sont à l'étude pour l'amélioration de notre sort et en particulier des retraites ouvrières. Mais qu'ils se dépêchent un peu MM. les députés d'en venir aux questions ouvrières afin que nos enfants au moins profitent d'une meilleure organisation sociale ! Ce qu'il nous recommande surtout, M. Chazette, c'est la solidarité entre les travailleurs ${ }^{188}$.

Toutefois, malgré ce témoignage élogieux, il semble que les cours de droit aient été un échec à l'Université populaire. Les juristes, très présents lors de la création de l'Université populaire, apparaissent de plus en plus marginalisés. Cet échec relatif peut s'expliquer par la volonté des juristes d'appréhender l'enseignement populaire du droit sans abandonner les stratégies corporatistes : attachement au positionnement doctrinal hétérodoxe et engagement social. À l'Université populaire, ces stratégies semblent propres aux juristes et ne s'observent pas dans la participation des autres universitaires - notamment des professeurs de lettres et de médecine - dont les cours rencontrent de vrais succès malgré un aspect technique.

L'expérience des cours de droit à l'Université populaire demeure malgré tout une expérience pédagogique intéressante qui questionne les méthodes d'enseignement du droit d'hier et d'aujourd'hui. Pour hier, des études sur l'enseignement du droit dans les 230 Universités populaires qui sont créées durant la Belle époque mériteraient d'être réalisées. Dans la région lyonnaise, d'autres universités populaires vont éclore avec l'Université populaire lyonnaise. En 1907, une éphémère université populaire d'obédience socialiste est créée à Lyon. Â Roanne, en 1901, des militants anarchistes assemblés par Jules Ravaté fondent une université populaire au sein de laquelle des cours de droit sont dispensés ${ }^{189}$. Surtout, à Villeurbanne, une Université populaire est créée par Georges Varaut après la Première Guerre mondiale. À Paris, la première Université populaire créée en 1896 - la Coopération des idées - mériterait l'intérêt des juristes ${ }^{190}$. Des cours de droit y sont dispensés par Ernest Tarbouriech ${ }^{191}$, Charles Gide, Pierre Baudouin et Lucien Le Foyer. De même, l'enseignement du droit de Saleilles au sein de l'Institut populaire du $5^{\mathrm{e}}$ arrondissement ${ }^{192}$ ou l'influence des cours de l'Université populaire La Semaille du $20^{\mathrm{e}}$ arrondissement sur le droit du chômage questionnent ${ }^{193}$. Pour aujourd'hui,

\footnotetext{
${ }^{187} A U P L, \mathrm{n}^{\circ} 4, \mathrm{p} .43$.

${ }^{188} A U P L, \mathrm{n}^{\circ} 3$, p. 106.

${ }^{189}$ L. Grelaud, «L’Université populaire de Roanne (1901-1907) », in Le mouvement social, $\mathrm{n}^{\circ}$ 35, 1961, p. 26-29. Les cours de droit sont donnés par Paul Renaison (alias Jean Ernest-Charles), docteur en droit et avocat, ainsi que par Albert Sérol, avocat, député socialiste en 1924, ministre du Travail en 1938 et Garde des Sceaux en 1940.

${ }^{190}$ On trouvera des indications dans La coopération des idées, journal populaire d'éducation et d'action sociale, journal de 1896 à 1914 et dans Les cahiers de l'Université populaire, revue de 1906 à 1907.

${ }^{191}$ F. Lekéal, « Ernest Tarbouriech : un juriste en socialisme. Itinéraire intellectuel », Cahiers Jean Jaurès, n 156 , 2001, p. 13-26. Tarbouriech enseigne ensuite au Collège libre des sciences sociales.

${ }^{192}$ R. Saleilles, Les nouvelles écoles du droit pénal, Paris, Rousseau, 1901, 29 p. et « Roman sociologique et roman social », rééd., in Revue Droit \& Littérature, n 3, 2019, p. 311-325.

${ }^{193} \mathrm{C}$. Bonici, L'indemnisation du chômage aux XIX et XX $X^{e}$ siècles à travers l'exemple lyonnais, thèse soutenue à l’Université Lyon III, 2010, p. 182.
} 
les juristes se sont emparés du mouvement de renaissance des Universités populaires initié au début des années 2000. À Lyon, cette mobilisation est significative avec la participation de professeurs de droit et de sciences politiques à la fondation de la nouvelle Université populaire de Lyon, à l'instar du Professeur Emmanuel Dockès ${ }^{194}$.

Pour hier comme pour aujourd'hui, les cours de droit aux universités populaires nous interrogent sur leur prétendue originalité et sur le caractère réellement populaire de ces enseignements. Si l'on ne peut douter des bonnes volontés des enseignants juristes, souvent hétérodoxes par rapport aux corps des Facultés de droit, on doit encore s'interroger, à notre époque de complexification du droit et d'enfermement de la doctrine sur elle-même, sur les principes qui fonderaient un enseignement du droit à destination d'un auditoire profane.

Jérôme Henning Professeur à l'Université Toulouse 1 Capitole

${ }^{194}$ E. Dockès et P. Corcuff, « Les principes de l'UPL » (https://unipoplyon.fr/principes-de-l-upl). 